\title{
Morphological and microarray analyses of human hepatocytes from xenogeneic host livers
}

\author{
Chise Tateno ${ }^{1,2,3}$, Fuyuki Miya ${ }^{4}$, Kenjiro Wake ${ }^{5,6}$, Miho Kataoka ${ }^{3}$, Yuji Ishida ${ }^{1,2}$, Chihiro Yamasaki ${ }^{1}$, Ami Yanagi ${ }^{1}$, \\ Masakazu Kakuni ${ }^{1}$, Eddie Wisse ${ }^{7}$, Fons Verheyen ${ }^{7}, K^{\prime}$ ouji Inoue ${ }^{8}, K^{2}$ ta Sato ${ }^{9}$, Atsushi Kudo ${ }^{9}$, Shigeki Arii ${ }^{9}$, \\ Toshiyuki Itamoto ${ }^{10}$, Toshimasa Asahara ${ }^{2,10}$, Tatsuhiko Tsunoda ${ }^{4}$ and Katsutoshi Yoshizato 1,2,3,11
}

We previously produced mice with human hepatocyte (h-hep) chimeric livers by transplanting h-heps into albumin enhancer/promoter-driven urokinase-type plasminogen activator-transgenic severe combined immunodeficient (SCID) mice with liver disease. The chimeric livers were constructed with h-heps, mouse hepatocytes, and mouse hepatic sinusoidal cells (m-HSCs). Here, we investigated the morphological features of the chimeric livers and the h-hep gene expression profiles in the xenogeneic animal body. To do so, we performed immunohistochemistry, morphometric analyses, and electron microscopic observations on chimeric mouse livers, and used microarray analyses to compare gene expression patterns in hepatocytes derived from chimeric mouse hepatocytes (c-heps) and h-heps. Morphometric analysis revealed that the ratio of hepatocytes to $\mathrm{m}$-HSCs in the chimeric mouse livers were twofold higher than those in the SCID mouse livers, corresponding to twin-cell plates in the chimeric mouse liver. The h-heps in the chimeric mouse did not show hypoxia even in the twin-cell plate structure, probably because of low oxygen consumption by the h-heps relative to the mouse hepatocytes (m-heps). Immunohistochemical and electron microscopic examinations revealed that the sinusoids in the chimeric mouse livers were normally constructed with h-heps and m-HSCs. However, a number of microvilli projected into the intercellular clefts on the lateral aspects of the hepatocytes, features typical of a growth phase. Microarray profiles indicated that $\sim 82 \%$ of 16605 probes were within a twofold range difference between $\mathrm{h}$-heps and c-heps. Cluster and principal component analyses showed that the gene expression patterns of c-heps were extremely similar to those of $\mathrm{h}$-heps. In conclusion, the chimeric mouse livers were normally reconstructed with $\mathrm{h}$-heps and $\mathrm{m}-\mathrm{HSCs}$, and expressed most human genes at levels similar to those in human livers, although the chimeric livers showed morphological characteristics typical of growth.

Laboratory Investigation (2013) 93, 54-71; doi:10.1038/labinvest.2012.158; published online 12 November 2012

KEYWORDS: human hepatocytes; microarray; ultrastructure; uPA/SCID mouse

The liver is a critical organ that can develop a number of serious diseases, including viral hepatitis, alcoholic liver disease, nonalcoholic liver disease, liver cirrhosis, and hepatocarcinoma. From a medical perspective, the liver is also consequential as it can metabolize drugs in the body. Because of differences in liver metabolic function between humans and experimental animals, the results in preclinical efficacy or safety studies using animals do not always apply to humans. On the other hand, although in vitro metabolism tests using human hepatocytes (h-heps) have been used to predict the metabolites of new drugs in humans, the results of these studies show limitations in predictivity. ${ }^{1}$ For investigating the mechanism of human liver disease and facilitating the development of medicines with high efficacy and safety for

\footnotetext{
${ }^{1}$ PhoenixBio Co., Ltd., Higashihiroshima, Japan; ${ }^{2}$ Hiroshima University Liver Research Project Center, Hiroshima, Japan; ${ }^{3}$ Yoshizato Project, Hiroshima Prefectural Institute of Industrial Science and Technology, Cooperative Link of Unique Science and Technology for Economy Revitalization (CLUSTER), Higashihiroshima, Japan; ${ }^{4}$ Laboratory for Medical Informatics, Center for Genomic Medicine, RIKEN, Yokohama, Japan; ${ }^{5}$ Liver Research Unit, Minophagen Pharmaceutical, Tokyo, Japan; ${ }^{6}$ Department of Anatomy, School of Dental Medicine, Tsurumi University, Yokohama, Japan; ${ }^{7}$ ELMI unit, Department of Molecular Biology, University Maastricht, Maastricht, The Netherlands; ${ }^{8}$ Institute of Electron Microscopy, School of Dental Medicine, Tsurumi University, Yokohama, Japan; ${ }^{9}$ Department of Hepatobiliary-Pancreatic Surgery, Graduate School of Medicine, Tokyo Medical and Dental University, Tokyo, Japan; ${ }^{10}$ Division of Frontier Medical Science, Department of Surgery, and Hiroshima University 21st Century COE Program for Advanced Radiation Casualty Medicine, Programs for Biomedical Research, Graduate School of Biomedical Sciences, Hiroshima University, Hiroshima, Japan and ${ }^{11}$ Developmental Biology Laboratory and Hiroshima University 21st Century COE Program for Advanced Radiation Casualty Medicine, Department of Biological Science, Graduate School of Science, Hiroshima University, Higashihiroshima, Japan Correspondence: $\operatorname{Dr} C$ Tateno, PhD, PhoenixBio Co., Ltd., 3-4-1 Kagamiyama, Higashihiroshima, Hiroshima 7390046, Japan. E-mail: chise.mukaidani@phoenixbio.co.jp 
humans, humanized animal models are needed, because they represent an approach that can circumvent the limitations of these other methods.

In a previous study, we transplanted h-heps into albumin enhancer/promoter-driven urokinase-transgenic severe combined immunodeficient (uPA/SCID) mice to create h-hepbearing (chimeric) mice. The host mouse hepatocytes (m-heps) in the livers of the chimeric mice were replaced with h-heps ${ }^{2}$ to the degree indicated by the replacement index (RI), which is the occupancy ratio of the h-hep area to the total (human and mouse) area on histological sections. In some cases, the RI in the mice was as high as $96 \%{ }^{2}$ The transplanted h-heps expressed mRNA for a variety of human drug-metabolizing enzymes and transporters, in a manner similar to that of the donor liver. ${ }^{2-4}$ The chimeric mouse livers were constructed with h-heps, m-heps, and mouse hepatic sinusoidal cells [m-HSCs; mainly Kupffer cells, sinusoidal endothelial cells (SECs), and stellate cells]. Moreover, the hepatocytes cooperated with the m-HSCs in liver function, ${ }^{5,6}$ with the $\mathrm{h}$-heps proliferating and functioning under the influence of the xenogeneic m-HSCs. However, few studies have investigated the structure of chimeric mouse livers ${ }^{7,8}$ or comprehensively analyzed their gene expression patterns.

The present study was undertaken to study the morphological, biochemical, and genetic similarities or differences between the livers of humans, chimeric mice, and control mice in order to verify whether chimeric mice can be considered to be a relevant model for biomedical experiments concerning the human liver.

\section{MATERIALS AND METHODS}

\section{Animals and Transplantation of H-heps}

This study was performed with the ethical approval of PhoenixBio, and the Hiroshima Prefectural Institute of Industrial Science and Technology Ethics Board. The uPA/ SCID mice were produced as previously described. ${ }^{2}$ All transplantation experiments used homozygotic uPA/SCID mice as hosts. Cryopreserved h-heps from a 9-month-old Caucasian boy (9MM) were purchased from In Vitro Technologies (Baltimore, MD, USA). Cryopreserved h-heps from a 5-year-old African-American boy (5YM), a 2-year-old Caucasian boy (2YM), a 4-year-old Caucasian girl (4YF), a 6-year-old African-American girl (6YF), and a 10-year old Caucasian girl (10YM) were purchased from BD Biosciences (San Jose, CA, USA). These h-heps were used as donor cells for chimeric mice.

For the transplantation study, 1 or 2 tubes of cryopreserved h-heps $\left(5-15 \times 10^{6}\right.$ cells/vial $)$ were thawed and transplanted into 10-50 uPA/SCID mice $\left(2.5-10.0 \times 10^{5}\right.$ viable cells/mouse). The human albumin (hAlb) concentration in the blood samples was periodically measured using latex agglutination immunonephelometry (LX Reagent 'Eiken' Alb II; Eiken Chemical, Tokyo, Japan) to predict the RI of h-heps in mouse livers. ${ }^{2}$ The hAlb concentration in mouse blood correlated well with the RI. Chimeric mice (Table 1) were selected from each transplantation study for immunohistochemistry and enzyme histochemistry, blood flow measurements, oxygen consumption studies, electron microscopic examination, blood biochemistry, microarray analysis, and real-time quantitative reverse transcriptionPCR (real-time qRT-PCR) analysis.

\section{Immunohistochemistry and Enzyme Histochemistry}

Frozen sections were prepared from the livers of 5YM-, 9MM-, and 2YM-chimeric mice (Table 1a, nos. 1-6), SCID mice, and humans (Table 1b). The sections were fixed in $-20^{\circ} \mathrm{C}$ acetone for $5 \mathrm{~min}$, and then incubated with anti-human cytokeratin (CK) 8/18 (h-hep marker protein), anti-mouse CK18 (m-hep marker protein), BM8 (a mouse macrophage-specific antigen), anti-mouse stabilin II (mouse liver endothelial marker protein), ${ }^{9}$ anti-desmin (stellate cell marker protein), anti-type IV collagen, anti-ZO-1, anticlaudin-1, and anti-occludin antibodies (tight junction proteins) (Table 2). The primary antibodies were visualized with Alexa 488- or 594-conjugated donkey anti-mouse-IgG, goat anti-rat IgG, or donkey anti-rabbit IgG (Invitrogen, Carlsbad, CA, USA) as secondary antibodies. The samples were then stained with Hoechst 33258 for nuclear staining. Human CK8/18 antibodies reacted with h-heps, but not m-heps; mouse CK18, BM8, and stabilin II antibodies reacted with $\mathrm{m}$-cells, but not h-cells. Other antibodies reacted with both $\mathrm{m}$ - and h-cells (Table 2). Dipeptidyl dipeptidase IV (DPPIV) enzyme histochemistry was performed on liver cryosections as previously shown. ${ }^{10}$

\section{Morphometric Analysis of Liver Cells}

Three 5YM-chimeric mice (Table 1a, nos. 1-3) and 3 SCID mice were used in this study. To identify hepatocytes, liver cryosections from chimeric and SCID mice were stained with human CK8/18 and mouse CK18 antibodies, respectively, as above. Frozen sections from both mice were stained with BM8, anti-mouse stabilin II, and desmin antibodies. The numbers of h-heps and m-heps in an arbitrary area were counted as human CK8/18- or mouse CK8-positive cells, respectively. The number of Kupffer cells, SECs, and stellate cells in an arbitrary area were counted as BM8-, mousestabilin II-, and desmin-positive cells, respectively. The number of h-heps or m-heps, mouse Kupffer cells, mouse SECs, and mouse stellate cells in a unit area were calculated, and the ratios of component cells in the chimeric and SCID mouse livers were expressed as circle graphs.

\section{Hypoxyprobe-1 treatment}

To determine whether h-heps in the chimeric mouse livers were hypoxic, uPA/SCID mice transplanted with h-heps (5YM) or m-heps were injected with $60 \mathrm{mg} / \mathrm{kg}$ body weight pimonidazole $\mathrm{HCl}$ from the Hypoxyprobe-1 kit (Hypoxyprobe, Burlington, MA, USA), at 3 and 2 weeks after transplantation, respectively. The livers were subsequently 
Table 1 Human liver tissues and chimeric mice used in this study

(a) Chimeric mice

\begin{tabular}{lcccccl}
\hline Donors & $n$ & Animal number (sex) & Weeks after transplantation & hAlb in blood (mg/ml) & Rl (\%) $)^{\text {a }}$ & Purpose \\
\hline 5YM & 3 & $1(\mathrm{M}), 2(\mathrm{M}), 3(\mathrm{~F})$ & $13-15$ & $12.2-16.5$ & $87-95$ & Morphometric analysis, immunohistochemistry \\
9MM & 2 & $4(\mathrm{M}), 5(\mathrm{~F})$ & 11 & $3.1,7.4$ & 53,75 & Immunohistochemistry, enzyme histochemistry \\
2YM & 1 & $6(\mathrm{~F})$ & 10 & 13.3 & 97 & Immunohistochemistry \\
5YM & 3 & $7-9(\mathrm{M})$ & 14 & $8.2-11.4$ & $75-85$ & ICG treatment \\
5YM & 3 & $10-12(\mathrm{M})$ & 10 & $8.5-9.6$ & $76-80$ & Microcirculation \\
5YM & 3 & $13(\mathrm{M}), 14(\mathrm{M}), 15(\mathrm{~F})$ & $11-14$ & $15.9-17.5$ & $94-97$ & Oxygen consumption \\
4YF & 2 & $16(\mathrm{~F}), 17(\mathrm{M})$ & 10,16 & $4.5,7.2$ & 75,74 & Transmission electron microscopy \\
10YM & 2 & $18(\mathrm{M}), 19(\mathrm{~F})$ & 8 & $5.1,4.9$ & 57,63 & Transmission electron microscopy \\
2YM & 2 & $20(\mathrm{M}), 21(\mathrm{~F})$ & 12,15 & $6.0-16.1$ & $70-95$ & Microarray, real-time qRT-PCR \\
9MM & 3 & $22-24(\mathrm{~F})$ & $10-14$ & $11.2-14.6$ & $91-100$ & Microarray \\
6YF & 3 & $25(\mathrm{~F}), 26(\mathrm{~F}), 27(\mathrm{M})$ & $11-12$ & $11.1 \pm 2.4$ & $81 \pm 6$ & Blood chemistry \\
5YM & 5 & $28-32(\mathrm{M})$ & $13 \pm 1$ & $8.0 \pm 1.3$ & $71 \pm 5$ & Blood chemistry \\
5YM & 5 & $33-37(\mathrm{~F})$ & $13 \pm 2$ & &
\end{tabular}

(b) Human liver tissues

\begin{tabular}{llll}
\hline Donors & Age (years) & Sex & Purpose \\
\hline $25 Y F$ & 25 & $F$ & Microarray, real-time qRT-PCR, Immunohistochemistry \\
$28 Y M$ & 28 & $M$ & Microarray, real-time $q R T-P C R$ \\
$57 Y M$ & 57 & $M$ & Microarray, real-time $q R T-P C R$ \\
$61 Y F$ & 61 & $F$ & Microarray, real-time $q R T-P C R$ \\
\hline
\end{tabular}

Abbreviations: F, female; hAlb, human blood albumin; M, male; RI, replacement index.

${ }^{\mathrm{a}} \mathrm{RI}$ calculated with blood hAlb levels using the formula of the correlation curve for each donor cell.

Table 2 Antibodies for immunohistochemistry

\begin{tabular}{|c|c|c|c|c|c|}
\hline Antibodies & Clone & Host & Specificity & Dilution & Supplier \\
\hline hCK8/18 & Monoclonal & Mouse & $\mathrm{h}$ & 100 & Cappel Laboratory, (Cochranville, PA, USA) \\
\hline BM8 & Monoclonal & Rat & $\mathrm{m}$ & 1000 & BMA Biomedicals, (Augst, Switzerland) \\
\hline mStabilin II & Monoclonal & Rat & $\mathrm{m}$ & 1000 & Gift from Dr Miyajima, (Tokyo University, Japan) \\
\hline Type IV collagen & Polyclonal & Rabbit & $h, m$ & 500 & LSL (Tokyo, Japan) \\
\hline ZO-1 & Polyclonal & Rabbit & $h, m$ & 50 & Zymed Laboratories (South San Francisco, CA, USA) \\
\hline Claudin-1 & Polyclonal & Rabbit & $h, m$ & 50 & Zymed Laboratories \\
\hline Occludin & Polyclonal & Rabbit & $h, m$ & 100 & Zymed Laboratories \\
\hline
\end{tabular}

Abbreviations: $h$, human specific; $m$, mouse specific.

harvested at $1 \mathrm{~h}$ after the injection. Chimeric mice (5YM, nos. 1-3) at 13-15 weeks after transplantation and 14-week-old SCID mice were also treated with pimonidazole $\mathrm{HCl}$ in the same manner. Acetone-fixed, frozen liver sections were treated with anti-pimonidazole adduct mouse monoclonal antibodies and biotin-conjugated anti-mouse IgG (Hypoxyprobe-1 kit) and diaminobenzidine according to the manufacture's protocol. 


\section{Blood flow measurements}

Indo-cyanine green (ICG, MP Biomedicals, LLC) dissolved in distilled water was injected into 3 chimeric mice (5YM, nos. $7-9)$ and 3 SCID mice via the tail vein at $25 \mathrm{mg} / \mathrm{kg}$ body weight. Blood was collected at $0,2,3,4,5,6,9,12,24$, and $60 \mathrm{~min}$ after the injection, and the ICG concentrations were measured in the sera by the microplate reader system Vmax (Molecular Device, Ontario, Canada). The blood flow was calculated by moment analysis.

The microcirculation of the livers in 3 chimeric (5YM, nos. 10-12) and 3 SCID mice was observed under anesthesia using intravital videomicroscopy, as described previously. ${ }^{11}$ For analyzing sinusoidal blood flow, at least 3 different sinusoids per mouse were captured in the microscopic fields at 500 frames/s using high-speed progressive videomicroscopy (HAS-LI, DITECT, Tokyo, Japan). This system enables highresolution $(1920 \times 1080)$ capture of real-time red blood cell flow in the sinusoid, with the hydrodynamics analyzed by the Flowsizer 2D software (DITECT).

\section{Measurement of Oxygen Consumption in H-Heps and M-Heps}

Chimeric mouse hepatocytes (c-heps) and m-heps were isolated by the collagenase perfusion method from three 5YM-chimeric mice (nos. 13-15) and three SCID mice, respectively, as below. Donor hepatocytes (5YM), c-heps, and $\mathrm{m}$-heps $\left(1.5 \times 10^{6}\right.$ cells $)$ were incubated in $5 \mathrm{ml}$ of $10 \mathrm{~mm}$ glucose in Dulbecco's modified Eagle's medium with $10 \%$ fetal bovine serum at $37^{\circ} \mathrm{C}$. Oxygen concentrations were measured every $5 \mathrm{~min}$ until $20 \mathrm{~min}$ using a SG6-ELK Professional dissolved oxygen meter (Mettler-Toledo KK, Tokyo, Japan). The oxygen consumption rate was calculated based on the oxygen concentration at each time point.

\section{Electron Microscopy}

The 4YF- and 10YM-chimeric mouse livers (Table 1a, nos. 16-19) were harvested under anesthesia. The mouse livers were prefixed by perfusing $1.5 \%$ glutaraldehyde in $0.1 \mathrm{M}$ cacodylate buffer $(\mathrm{pH} 7.4)$ through a portal vein at $4{ }^{\circ} \mathrm{C}$ for $40 \mathrm{~s}$ using a syringe. The livers were cut into $1-\mathrm{mm}^{3}$ blocks with a razor blade under the affluent solution in $2 \%$ glutaraldehyde/0.1 M cacodylate buffer $(\mathrm{pH}$ 7.4). Next, the sample blocks were postfixed with $1 \% \mathrm{OsO}_{4} / 0.1 \mathrm{M}$ phosphate buffer ( $\mathrm{pH}$ 7.4) for $1 \mathrm{~h}$ at $4{ }^{\circ} \mathrm{C}$. After the blocks were washed with $0.1 \mathrm{M}$ phosphate buffer ( $\mathrm{pH}$ 7.4), they were dehydrated through an ethanol series and propylene oxide, and embedded in Epon. ${ }^{12}$ Ultrathin sections were cut with a Reichert Ultracut ultramicrotome (Reichert Optikwork, Vienna, Austria), stained with lead and uranyl salts, and examined in a JEOL-100 CX TEM (Tokyo, Japan) operated at $80 \mathrm{kV}$.

For scanning electron microscopy (SEM), the livers of 2YM-chimeric mice (Table 1a, nos. 20 and 21) were perfused through a portal vein with filtered $1.5 \%$ glutaraldehyde under anesthesia, and then with $1 \%$ sucrose $/ 0.067 \mathrm{M}$ cacodylate buffer ( $\mathrm{pH}$ 7.4) at room temperature for $5 \mathrm{~min} .{ }^{13}$ The livers were cut into $1 \times 1 \times 5 \mathrm{~mm}$ blocks with a razor blade under the fixative solution, and washed with $1 \%$ sucrose $/ 0.067 \mathrm{M}$ cacodylate buffer ( $\mathrm{pH}$ 7.4). The blocks were postfixed with $1 \% \mathrm{OsO}_{4} / 0.1 \mathrm{M}$ phosphate buffer ( $\mathrm{pH} 7.4$ ) for $1 \mathrm{~h}$ at $4{ }^{\circ} \mathrm{C}$, and then washed with $0.1 \mathrm{M}$ phosphate buffer ( $\mathrm{pH}$ 7.4). They were dehydrated with an ethanol series. After being treated with $100 \%$ ethanol, the samples were immersed for $10 \mathrm{~min}$ in $100 \%$ hexamethyldisilazane (Sigma Chemical), and dried in a desiccator. The samples were then mounted on stubs and sputter-coated with $10 \mathrm{~nm}$ of gold. They were observed under an SEM (model XL30, Philips).

\section{Isolation of H-Heps from Human and Chimeric Mouse Livers for Microarray and Real-Time qRT-PCR Analyses} Livers were obtained from 4 individuals: 2 men aged 28 and 57 years (28YM and $57 \mathrm{YM}$, respectively) and 2 women aged 25 and 61 years (25YF and 61YF, respectively; Table $1 \mathrm{~b}$ ). The donors gave informed consent before surgery, according to the 1975 Declaration of Helsinki. The h-heps were isolated from these tissues, as previously reported, ${ }^{2}$ and subjected to microarray and real-time qRT-PCR analyses (Table 1b). The c-heps were isolated from 9MM-chimeric mice (Table 1a, nos. 22-24) 10-14 weeks after transplantation and 6YF-chimeric mice (Table 1a, nos. 25-27) 11-12 weeks after transplantation. These animals had high $(>70 \%)$ RI levels (Table 1a). The livers were disaggregated using the two-step collagenase perfusion method: ${ }^{14}$ the livers were perfused for $20 \mathrm{~min}$, and then centrifuged 3 times at $50 \mathrm{~g}$ for $2 \mathrm{~min}$. The hepatocytes obtained as pellets from the c-heps were used for the microarray and real-time qRT-PCR analyses. The cells were frozen in liquid nitrogen and stored in a deep freezer until total RNA isolation.

\section{Microarray Analysis}

Microarray-based gene expression analysis was performed at the hepatocyte level using c-heps or h-heps as RNA sources. Four human individuals (25YF, 28YM, 57YM, and 61YF; Table 1b) and 6 chimeric mice (Table 1a, nos. 22-27) were used in the microarray assay.

Total RNA was isolated from each hepatocyte sample using TRIzol reagent (Invitrogen), treated with DNase (Qiagen), and purified using the RNase-Free DNase Set (Qiagen) and the RNeasy Mini Kit (Qiagen). The RNAs from c-heps or h-heps were applied to the microarray assay using GeneChip Human Genome U-133 Plus 2.0 Array (Affymetrix, Santa Clara, CA, USA) with 54675 probe sets according to the manufacturer's instructions. The obtained mRNA expression profiles (c-heps, $n=6$; h-heps, $n=4$ ) are designated as the hepatocyte-level profiles in this study. The gene expression array data were normalized using the MAS5 algorithm (Affymetrix). The signal reliability of each probe was determined based on the MAS5 Call algorithm (Affymetrix), and each probe was assigned to 1 of 3 flags ( $\mathrm{P}$, present; $\mathrm{M}$, marginal; and A, absent). For correcting bias between chips, quantile normalization ${ }^{15}$ was applied to all array data using 
R software. We deposited our array data to NCBI GEO (Gene Expression Omnibus, http://www.ncbi.nlm.nih.gov/geo/, GEO ID GSE33846, GSE18674).

For determining the similarity of c-heps and h-heps, the microarray data of c-heps, h-heps, and 22 human tissues (h-tissues) (liver, kidney, pancreas, cerebellum, cortex, fetal brain, spinal cord, bone marrow, heart, skeletal muscle, salivary gland, colon, stomach, small intestine, lung, uterus, prostate, thyroid, trachea, spleen, thymus, and testis) were analyzed using principle component analysis (PCA) for 46336 probes that were assigned as positive ( $\mathrm{P}$ flag) for at least one of the flags in any of c-heps, h-heps, or 22 h-tissues, including h-liver. PCA and cluster analysis were performed using GeneSpring GX 11.0 (Agilent Technologies) and R software.

Liver signature probes were selected as follows: 685 and 805 probes with expression levels twofold higher or lower in the h-liver than in all of the other $21 \mathrm{~h}$-tissues were selected among the 54675 probes as liver high signature probes (Supplementary Table 1) and liver low signature probes (data not shown), respectively. The results of Gene Ontology (GO) and pathway analysis indicated that the selected 685 probes represent liver-specific components (Supplementary Table 2). Cluster analysis was performed with the average linkage method and Euclidian distance using Cluster and TreeView. ${ }^{16}$

\section{Determination of mRNA Expression Levels by Real-Time qRT-PCR Analysis}

A total of 17 human genes (IGF-1, SOCS2, NNMT, IGFLS, KLOTHO, P4AH1, SLC16A1, SRD5A1, SCD, FADS1, FADS2, FASN, DGAT2, ADPN, SREBP1c, FABP, and AKR1B10) were selected based on a previous study. ${ }^{17}$ That is, these genes were previously determined to be up- or down-regulated in chimeric mouse livers when chimeric mice were administered human growth hormone (hGH). ${ }^{17}$ The mRNA expression levels of these genes were quantified by real-time qRT-PCR. The specimens used for the microarray analysis were used for the extraction of total RNA, which was performed as described above. cDNA was synthesized using $1 \mu \mathrm{g}$ of RNA, PowerScript reverse transcriptase (Clontech, Mountain View, CA, USA), and oligo-dT primers (Invitrogen) according to the manufacturer's instructions, and then subjected to real-time qRT-PCR. Genes were amplified with a set of genespecific primers ${ }^{17}$ and SYBR Green PCR mix in a PRISM 7700 Sequence Detector (Applied Biosystems, Tokyo, Japan). We confirmed conditions consisted of an initial denaturation step at $95^{\circ} \mathrm{C}$ for $10 \mathrm{~min}$, followed by 40 cycles at $95^{\circ} \mathrm{C}$ for $15 \mathrm{~s}$, and $60^{\circ} \mathrm{C}$ for $1 \mathrm{~min}$. All data were calculated by the comparative threshold cycle $(\mathrm{Ct})$ method as previously described. ${ }^{18}$ Contamination by m-heps did not affect the RT-PCR determination of human gene expression because the expression level of each gene was normalized against the human glyceraldehyde 3-phosphate dehydrogenase (hGAPDH) gene.

\section{Gene Enrichment Analysis}

Gene and GO information was collected from NCBI build 37 (http://ncbi.nlm.nih.gov) and the GO (http://www. geneontology.org) sites, respectively. Pathway information was collected from KEGG (http://www.genome.jp/kegg) and the Ingenuity Pathways Analysis (IPA) software (Ingenuity Systems). The gene enrichment analysis was performed using only GO and the pathway group when at least two or more genes were assigned.

\section{Biochemical Tests}

Blood was collected from the inferior vena cava of 5 male and 5 female 5YM-chimeric mice (Table 1a, nos. 28-37), 5 male uPA/SCID mice, and 3 male SCID mice at the time of killing. The sera were used for the following biochemical tests: glutamic pyruvic transaminase (GPT), glutamic oxaloacetic transaminase (GOT), $\gamma$-glutamyltransferase (GGT), cholinesterase (CHE), blood urea nitrogen (BUN), total cholesterol (TCHO), high-density lipoprotein cholesterol (HDL-c), triglycerides (TGs), total bilirubin (TBIL), glucose (GLU), total albumin (ALB), and total protein (TP). These tests were conducted using a Fuji Drichem $3500 \mathrm{~V}$ serum analyzer (Fujifilm, Tokyo, Japan).

\section{Statistical Analyses}

Microarray data were evaluated by Welch's $t$-test (two-sided) and adjusted for multiple testing using the BenjaminiHochberg (B-H) false discovery rate (FDR). ${ }^{19}$ The gene enrichment analysis and the significance of overlap between two groups of transcripts were performed using Fisher's exact test, and the gene enrichment analysis was corrected with the B-H FDR. ${ }^{19}$ Data obtained in blood chemistry were analyzed among groups by ANOVA. When the overall F-statistics were significant, significance was determined by Sheffe's test with significance level $\alpha=0.05$.

\section{RESULTS \\ Construction of Chimeric Mouse Livers with H-Heps and M-HSCs}

Cryopreserved donor hepatocytes (5YM) were thawed and transplanted into 54 2-4-week-old uPA/SCID mice $\left(2.5 \times 10^{5}\right.$ cells/animal), and blood hAlb concentrations were monitored (Figure 1a). In all, 50 mice remained alive beyond 10 weeks of age. Of these, 1,3 , and 2 mice showed $1-3,3-5$, and $5-7 \mathrm{mg} / \mathrm{ml} \mathrm{hAlb}$ blood concentrations, respectively, at 10 weeks of age. Also, 44 mice secreted $>7 \mathrm{mg} / \mathrm{ml} \mathrm{hAlb}$ into the blood, and 6 mice died after reaching 10 weeks of age $(5-7 \mathrm{mg} / \mathrm{ml}, n=1 ;>7 \mathrm{mg} / \mathrm{ml} \mathrm{hAlb}, n=5)$. Three mice showing $>7 \mathrm{mg} / \mathrm{ml} \mathrm{hAlb}$ were used for morphometric analysis (nos. 1-3, Figure $1 \mathrm{~b}$ and $\mathrm{c}$ ). Immunohistochemistry showed that the hepatocytes and the nuclei were smaller in the chimeric mice than in the SCID mice (Figure 2a and b), which were similar to the h-heps in a human body (data not shown). BM8-positive mouse Kupffer cells (Figure $2 \mathrm{c}$ and d), desmin-positive mouse stellate cells (Figure $2 \mathrm{~g}$ and $\mathrm{h}$ ), and 
$\mathrm{m}$-Stabilin II-positive m-SECs (Figure 2e and f) were uniformly distributed in both livers, but their densities were lower in the chimeric mice than in the SCID mice. Desminpositive mouse stellate cells with cytoplasmic projections were closely associated with the m-SECs (data not shown).
The BM8-positive mouse Kupffer cells were smaller in the chimeric mice $\left(39.2 \pm 4.3 \mu \mathrm{m}^{2}\right)$ than in the SCID mice $\left(50.6 \pm 4.0 \mu \mathrm{m}^{2}\right.$; Figure $2 \mathrm{c}$ and $\left.\mathrm{d}\right)$. Moreover, large BM8-positive cells were occasionally present near portal veins in the chimeric mice (data not shown).

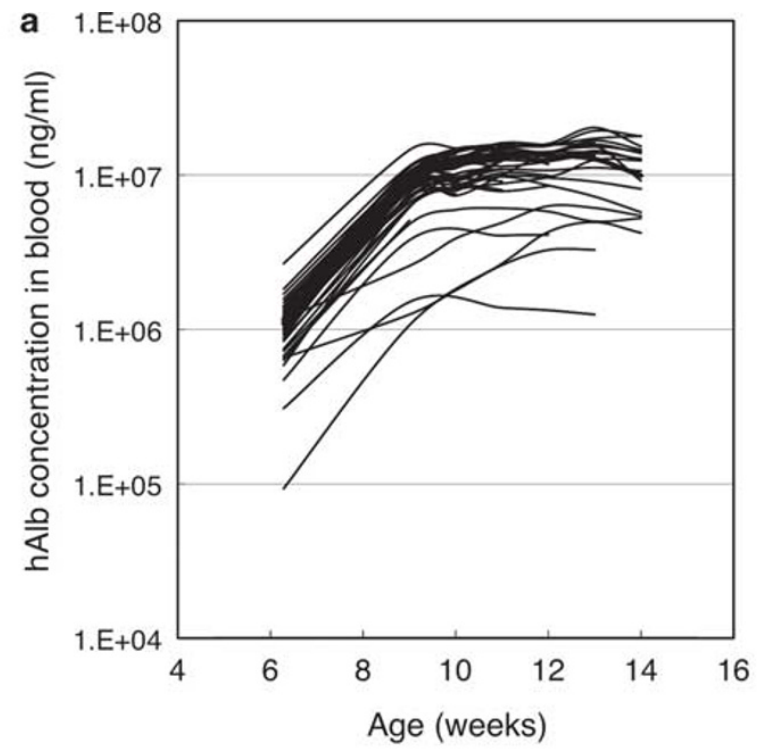

b

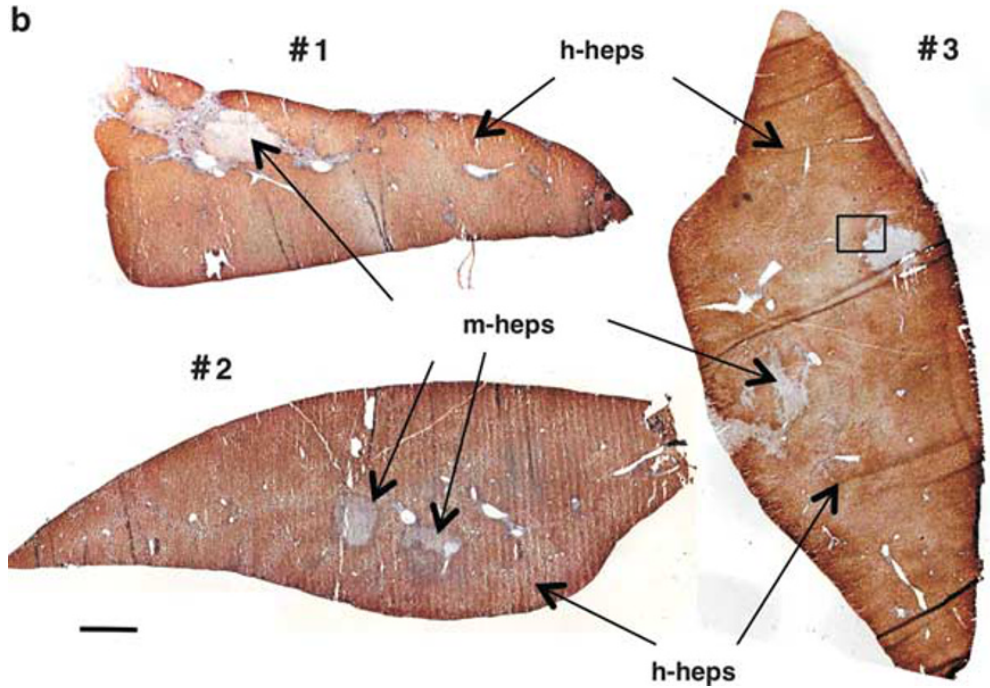

C

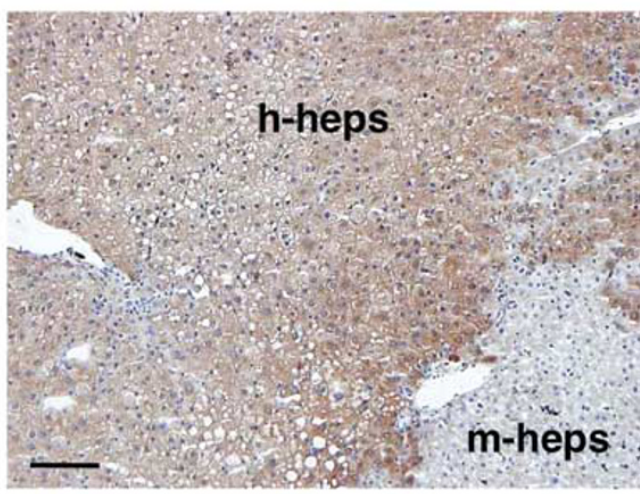

Figure 1 Changes in hAlb concentration in the blood and immunostained livers of mice transplanted with h-heps. (a) The graph shows an example of the results of the transplantation of h-heps into uPA/SCID mice. The h-heps from 5YM were transplanted into 54 uPA/SCID mice, and the hAlb concentrations in mouse blood were monitored. A total of 50 mice remained alive beyond 10 weeks of age. Of these, 1, 3, and 2 mice showed 1-3, $3-5$, and $5-7 \mathrm{mg} / \mathrm{ml}$ hAlb concentrations, respectively, after 10 weeks of age. Forty-four mice leached $>7 \mathrm{mg} / \mathrm{ml}$ hAlb into the blood, which corresponds to $>70 \%$ RI. Three 5 YM-chimeric mice were used for morphometric analysis. (b) The livers of three 5YM-chimeric mice (nos. 1-3) were immunostained with human CK8/18 antibodies. The brown-colored area is that of h-heps. The mouse livers were almost entirely repopulated by h-heps. Bar $=1 \mathrm{~mm}$. (c) A magnified view of the liver region enclosed by the square for chimeric mouse no. 3 of (b). Bar $=100 \mu \mathrm{m}$.

Figure 2 Immunohistochemistry of SCID and chimeric mouse liver for morphometric analysis. Three SCID (a, c, e, g) and 5YM-chimeric mouse $(\mathbf{b}, \mathbf{d}, \mathbf{f}, \mathbf{h})$ livers were stained with Hoechst 33248 and m-CK18 (a) or h-CK8/18 (b); BM8 (c, d); anti-mouse stabilin II (e, f); desmin and m-CK18 (g) or h-CK8/18 (h). The cells and nuclei in the SCID mice were larger than those in the chimeric mice (a, $\mathbf{b})$. The densities of BM8-, anti-mouse stabilin II-, and desmin-positive cells were lower in the chimeric mice than those in the SCID mice (c-h). BM8-positive cells were smaller in the chimeric mice than those in the SCID mice (c, d). Magnification is identical for panels belonging to the same horizontal pair. Bar $=100 \mu \mathrm{m}$. 

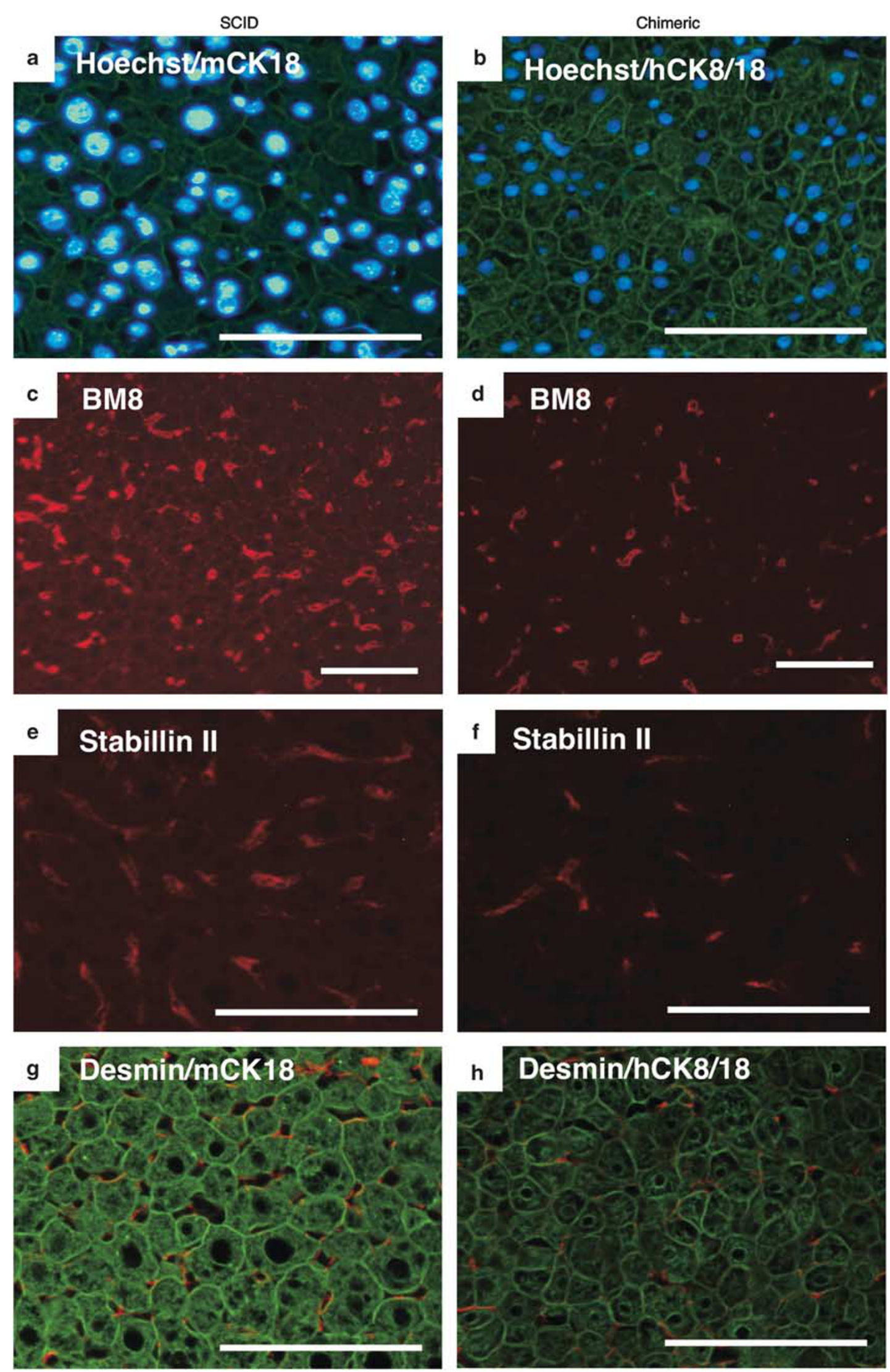

Figure 2 For caption please refer page 59. 


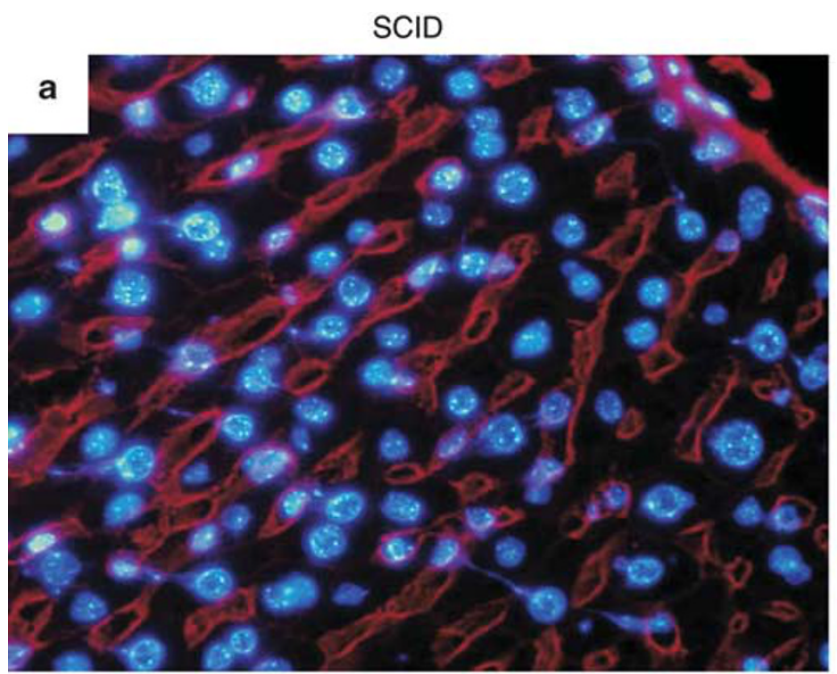

Hoechst/typelV collagen

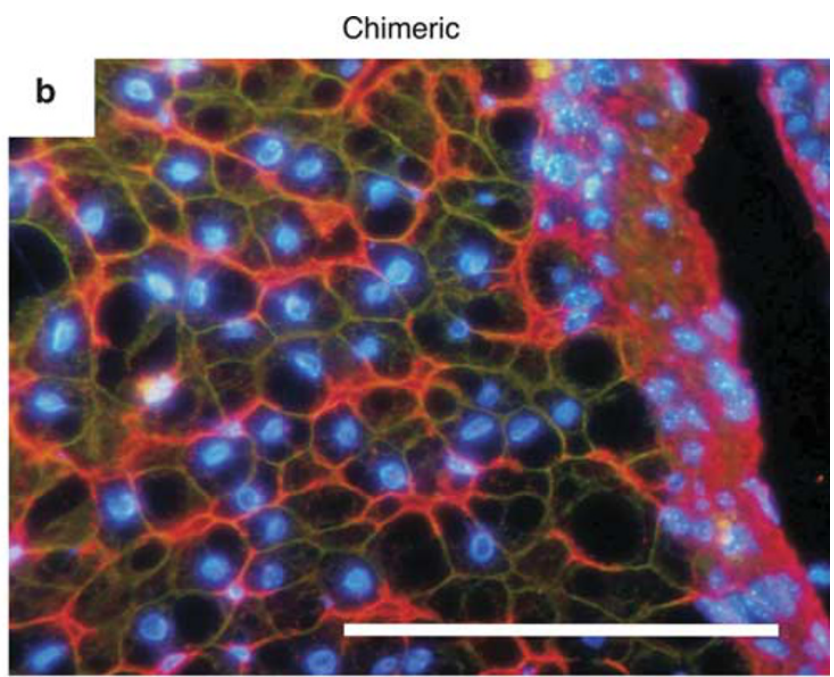

Hoechst/hCK8/18/typeIV collagen

C

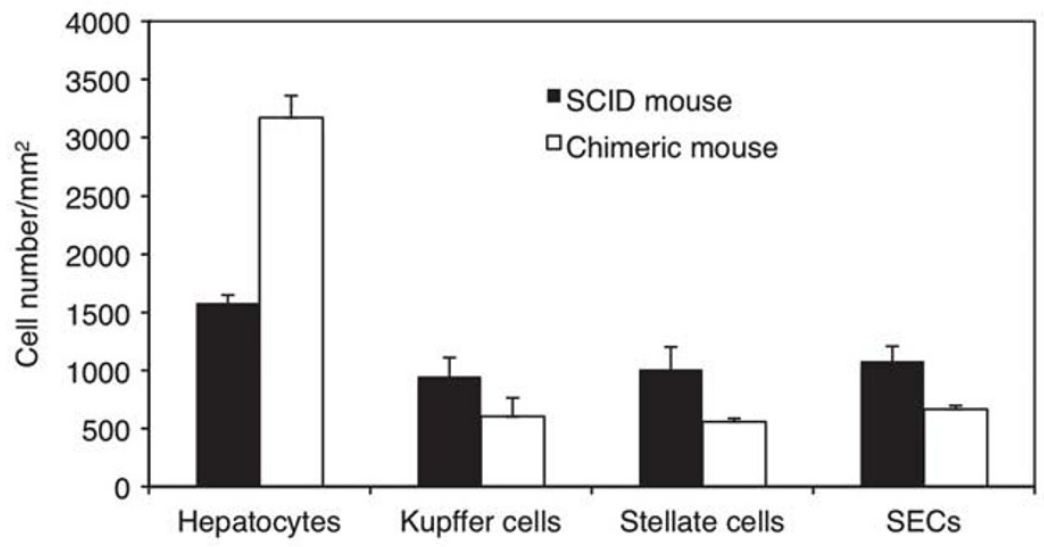

d

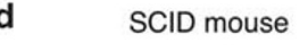

e Chimeric mouse
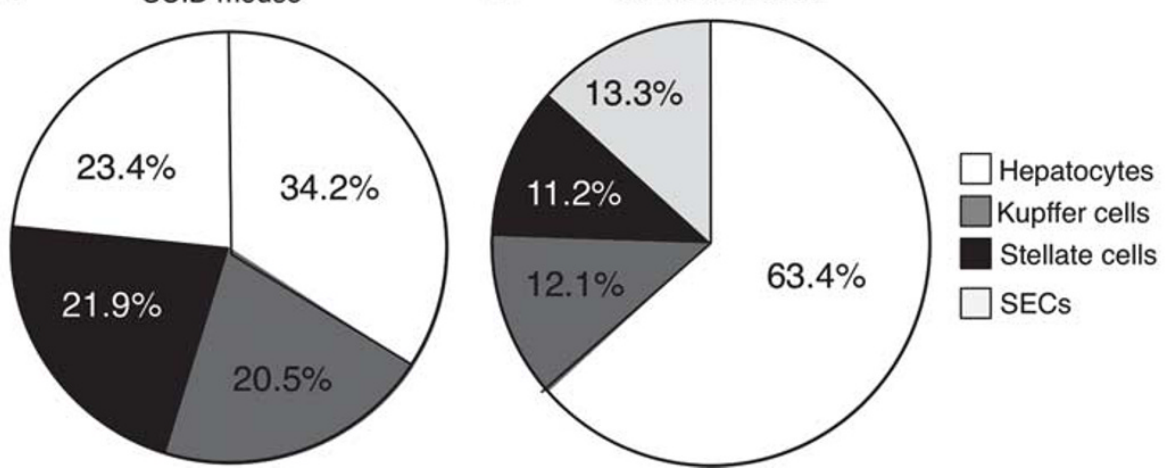

Figure 3 Immunohistochemistry of chimeric mouse livers stained for Type IV collagen, and the ratio of component cells in SCID (a) and chimeric (b) mouse livers. SCID and 5YM-chimeric mouse livers were stained with Hoechst and anti-type IV collagen antibody. 5YM-chimeric mouse livers were additionally stained with human CK8/18 antibodies. SCID and chimeric mouse livers showed single-cell and twin-cell plates, respectively. Bar $=100 \mu \mathrm{m}$. The number of hep cells, BM8-positive cells (Kupffer cells), desmin-positive cells (stellate cells), and anti-mouse stabilin II-positive cells (SECs) were counted under a microscope, and the density of each cell type was calculated (c). The ratios of the component cells in SCID (d) and chimeric mice (e) were calculated from the data of (c), and expressed as circle graphs. The ratios of Kupffer cells, stellate cells, and SECs were similar in the SCID and chimeric mice, but the ratio of hepatocytes to HSCs (Kupffer cells, stellate cells, and SEC) was twofold greater in the chimeric mice than that in the SCID mice.

Type IV collagen depositions were observed on the basal aspect of the heps (Figure $3 \mathrm{a}$ and $\mathrm{b}$ ). In the SCID mouse liver, the $\mathrm{m}$-heps showed single-cell plates (Figure 3a), whereas the h-heps in the chimeric mouse liver showed twin-cell plates (Figure $3 \mathrm{~b}$ ). The h-heps or m-heps in combination with the m-HSCs (m-Kupffer cells, m-SECs, and m-stellate cells) 
were counted in an arbitrary area on samples from three chimeric mice or three SCID mice, respectively (Figure 3c), and the ratios were subsequently compared (Figure 3d and e). The ratios of the Kupffer cells, SECs, and stellate cells were similar between groups. However, the ratios of the $\mathrm{h}$-heps and m-HSCs in the chimeric mice were twofold greater than those in the SCID mice (Figure 3d and e).

\section{Lack of Hypoxia in H-Heps with Twin-Cell Plates}

To determine whether the h-heps with the twin-cell plates were in a hypoxic state, the chimeric mice were treated with hypoxyprobe- 1 . The result revealed that the m-heps in the 14-week-old SCID mouse liver (Figure 4a) and the h-heps in the chimeric mouse liver at 13-15 weeks after transplantation (growth termination phase, Figure $4 \mathrm{~b}$ ) did not show hypoxia. The growing $\mathrm{m}$-heps at 2 weeks after transplantation in the uPA/SCID mouse liver showed hypoxia (Figure 4c), whereas the growing h-heps at 3 weeks after transplantation did not show hypoxia (Figure 4d). The host uPA/SCID mouse hepatocytes (Figure $4 \mathrm{c}$ and d) were damaged and atrophic because of the expression of uPA. Thus, our results suggest that the damaged uPA/SCID mouse liver might be hypoxic.

As determined by measuring ICG concentrations, the liver blood flow was similar between the SCID and chimeric mice (Figure 4e and $\mathrm{f}$ ), suggesting that the overall blood flow within the liver was similar between these two groups of mice. The livers of the chimeric mice were enlarged, with the liver weight to body weight ratio at $13.0 \pm 0.3 \%$ for the chimeric mice and at $5.4 \pm 0.5 \%$ for the SCID mice. ${ }^{20}$ The microcirculation of the SCID and chimeric mice livers were then determined in order to investigate the effects of the enlarged liver size on sinusoidal blood flow in the chimeric mouse liver. The blood cell flow rate was slower in the sinusoids of the chimeric mouse liver $(0.0116 \pm 0.003 \mu \mathrm{m} / \mu \mathrm{s}$, Figure $4 \mathrm{~g}$ ) than in those of the SCID mouse liver $(0.0247 \pm 0.005 \mu \mathrm{m} / \mu \mathrm{s}$, Figure $4 \mathrm{~g})$. In addition, no disturbances were observed in the microcirculation of either the SCID or chimeric mice livers, despite the blood flow in the chimeric mouse liver occurring at half the rate seen in the SCID mouse liver.

The oxygen consumption of the c-heps increased in a cell number-dependent manner (Figure $4 \mathrm{~h}$ ). Moreover, the oxygen consumption of $1.5 \times 10^{6} \mathrm{~m}$-heps was higher than that of $5.0 \times 10^{6} \mathrm{c}$-heps (Figure $4 \mathrm{~h}$ ). The oxygen consumption rates of $\mathrm{m}$-heps from SCID mice, h-heps from chimeric mice, and donor h-heps from the chimeric mice were $4.82 \pm 0.25$, $1.07 \pm 0.13$, and $0.54 \mu \mathrm{m} / \mathrm{ml} / 10^{6}$ cells, respectively (Figure $4 \mathrm{i}$ ).

\section{Ultrastructure of Chimeric Mouse Livers}

The chimeric mouse livers were found to be composed of three visually identifiable regions of different colors. ${ }^{1}$ The white and red regions corresponded to the original diseased $\mathrm{m}$-heps and uPA gene-deleted $\mathrm{m}$-hep regions, respectively. The medium-toned regions between the white and red regions corresponded to h-heps. Electron microscopic observation showed that the h-heps in the medium-colored regions were distinguished as cells with abundant glycogen and large lipid droplets in the cytoplasm (Figure 5a). In contrast, abundant small granules $(0.1-0.5 \mu \mathrm{m}$ in diameter) were observed in the original diseased $\mathrm{m}$-hep cells in the white regions (Figure 5b). M-heps in the red regions showed a normal morphology (Figure $5 \mathrm{c}$ ).

The space of Disse was observed between the h-heps and the SECs of the mouse liver (Figure 5a), whereas bile canaliculi were seen between the h-heps (Figure 5d). In the space of Disse, stellate cells with lipid droplets were observed (Figure 5e). Meanwhile, SEM revealed fenestrae in the SECs (Figure 5f). The h-heps adhered to each other and formed bile canaliculi on the apical surfaces, whereas their basal surfaces had many microvilli facing the thin mouse SECs (Figure $5 \mathrm{a}$ and $\mathrm{d}$ ). These results indicate normal reconstruction of the chimeric mouse livers with h-heps and m-HSCs.

\section{Cell-Cell Adhesion Between Hepatocytes}

In chimeric mouse livers, immunocytochemistry detected tight junction proteins, such as ZO-1 (Figure 6a), claudin-1 (data not shown), and occludin-1 (Figure 6c), on membranes adjacent to h-heps, as would occur in the human liver (Figure $6 \mathrm{~b}$ and d). However, the frequency was lower in the chimeric mouse livers than in the human ones. DPPIV activity was observed on the microvilli of bile canaliculi (Figure 6f). In the chimeric mouse livers, DPPIV enzyme activity was concentrated on membranes adjacent to the h-heps. Interestingly, faint signals were detected on the h-hep cell surfaces near portal veins in the chimeric livers (Figure 6e).

Bile canaliculi are organized by junctional complexes. Electron microscopic examination demonstrated that junctional complexes, consisting of tight junctions, adherence

\footnotetext{
Figure 4 Analyses of pimonidazole binding (a-d), blood flow (e-g), and oxygen consumption (h, i) in SCID and chimeric mouse livers. SCID and chimeric mice and UPA/SCID mice transplanted with m-heps ( 2 weeks after transplantation) and h-heps ( 3 weeks after transplantation) were injected with pimonidazole $\mathrm{HCl}$; the livers were then immunostained for pimonidazole adducts. SCID (a) and chimeric mouse livers (b) were negative for the adducts, whereas UPA/SCID mouse hepatocytes were positive for pimonidazole adducts (c, d). M-hep-colonies (c, arrowheads) were positive, whereas h-hep-colonies were negative (d, arrowheads). Bar $=100 \mu \mathrm{m}$. The chimeric mice and SCID mice were injected with ICG. The ICG concentrations in the mouse sera were subsequently monitored (e). The clearance rate of ICG was similar between the SCID and chimeric mice (f). The sinusoidal blood flow in the chimeric mouse liver was approximately one-half of that in the SCID mouse liver (g). The oxygen consumption of c-heps and m-heps was monitored (h). The oxygen consumption of c-heps was $1 / 4.5$ lower than that of $m$-heps. ${ }^{*} P<0.05,{ }^{* *} P<0.01$.
} 

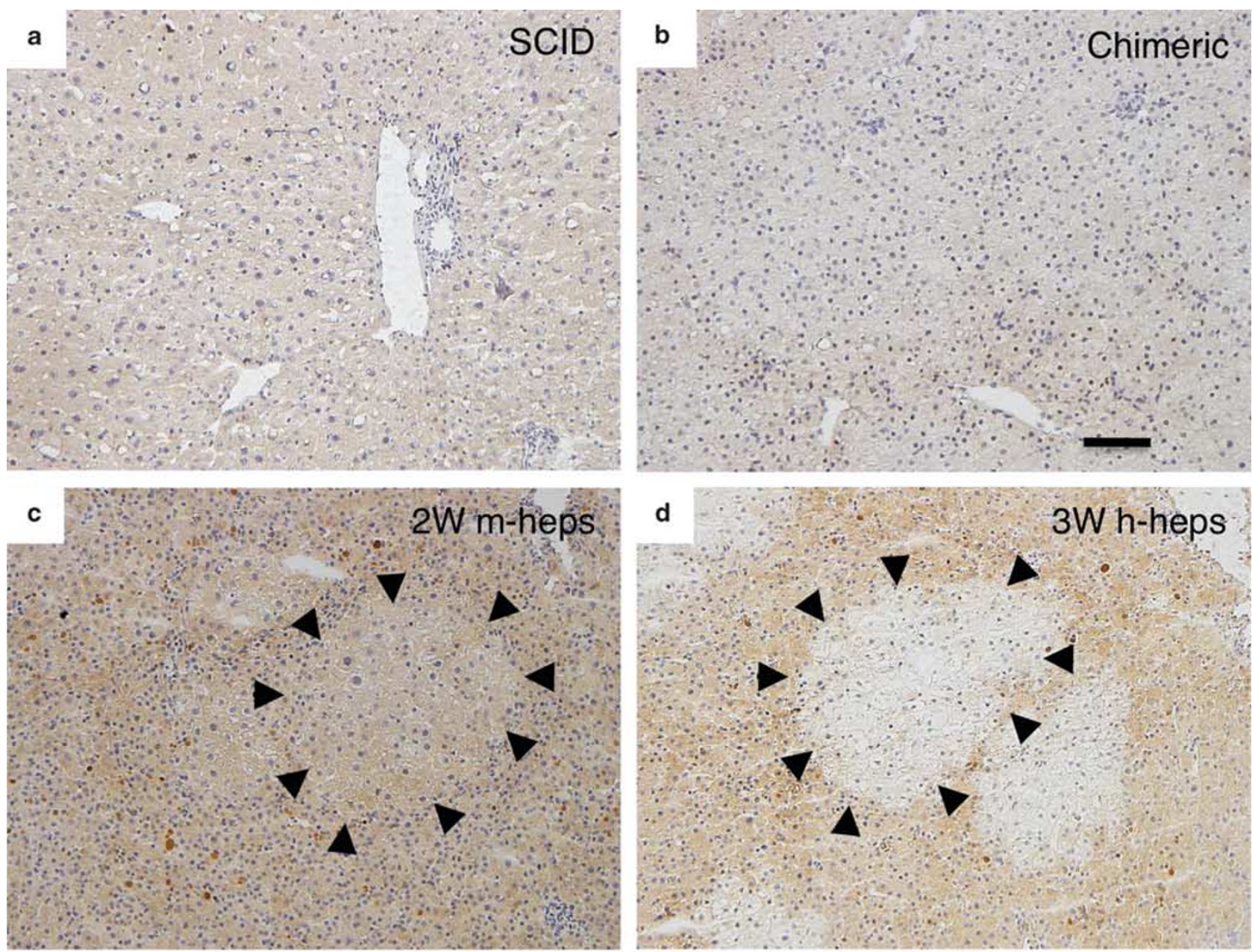
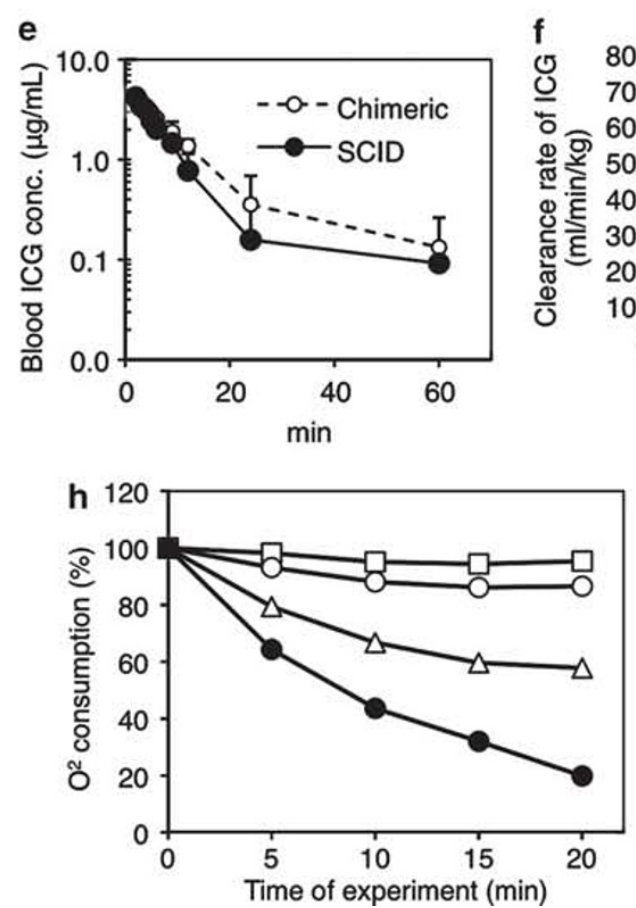
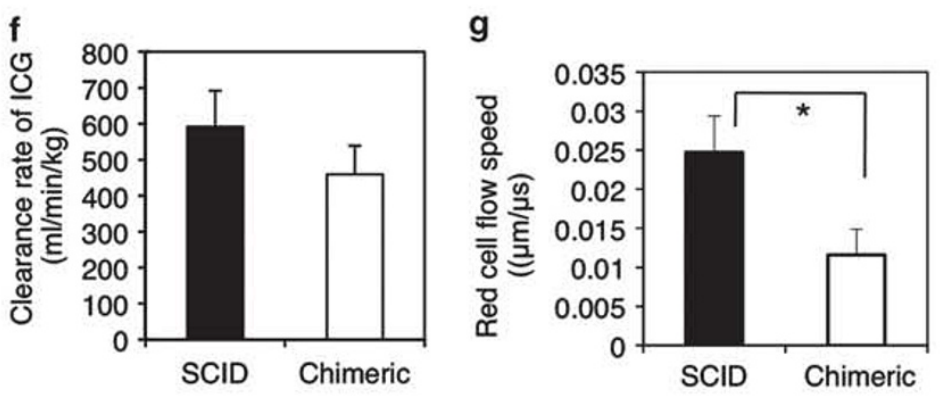

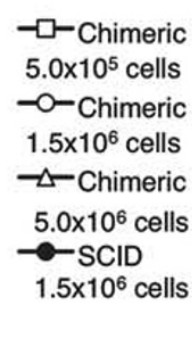

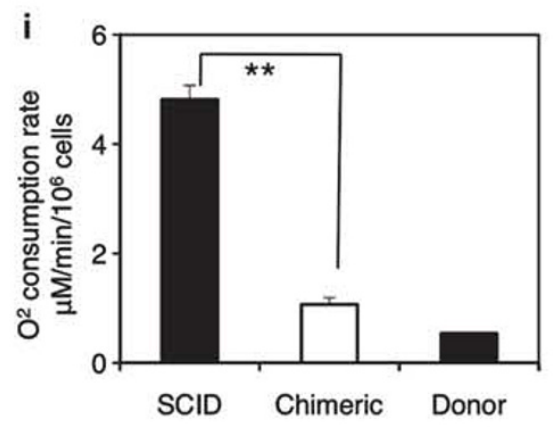


junctions, and desmosomes, directly adjoined bile canaliculi between h-heps (Figure 7a). In rare cases, bile canaliculi were also formed between h-heps and m-heps (Figure $7 \mathrm{~b}$ ). In the peripheral areas of the cytoplasm in groupings of two h-heps and one $\mathrm{m}$-hep, many microvilli projected into the intercellular clefts on the lateral aspects of the hepatocytes (Figure 7c). A small number of cone-like cytoplasmic processes formed contacts with neighboring cells, not only between h-heps (Figure 7c) but also between m-heps and h-heps (Figure 7d). Electron microscopic observation showed gap junctions between h-heps (Figure 7e) and between m-heps (data not shown).

\section{Changes in Blood Biochemistry by Repopulation of Mouse Liver with H-Heps}

Sera of the chimeric (Table 1a), uPA/SCID, and SCID mice were biochemically tested (Table 3 ). The data for the normal range in humans ${ }^{21,22}$ are also shown in Table 3. GOT and GPT were higher in the chimeric and uPA/SCID mice than in the normal mice because of the liver damage by uPA expression in the m-heps. ${ }^{2}$ GGT was significantly higher in the chimeric mice than in the SCID mice and the normal human samples. ${ }^{21}$ CHE activity differed between humans and male SCID mice $\left(660-1620 \mathrm{IU} / \mathrm{l}^{22}\right.$ and $18 \mathrm{IU} / \mathrm{l}$, respectively). CHE activity in the male chimeric mice was $\sim 400 \mathrm{IU} / \mathrm{l}$. These data indicate that the c-heps synthesized and secreted GGT and CHE in the same manner as human hepatocytes. HDL-c and low-density lipoprotein cholesterol (LDL-c) ratios also differed between humans and mice. HDLc was higher than LDL-c in the SCID mice, whereas LDL-c was higher than HDL-c in humans. ${ }^{22}$ LDL-c was higher than HDL-c in the chimeric mice (data not shown), but the HDL$c$ value was lower than that of normal humans (Table 3). ALB was higher in humans than in mice. Similarly, ALB was higher in the chimeric mice than in the SCID mice, indicating that the sera in the chimeric mice may have been acquiring human characteristics. The biomarkers BUN, TCHO, TG, TBIL, GLU, and TP did not significantly differ between male chimeric and male SCID mice.

\section{Comparison of Gene Expression Profiles in Hepatocytes Isolated from Chimeric Mouse and Human Livers}

The mRNA expression profiles of c-heps and h-heps were compared. The c-heps were isolated from three 9MM- and 6YF-chimeric mice (Table 1a), whereas h-heps were isolated from four human liver tissues (Table $1 \mathrm{~b}$ ). The gene profiles were determined for the two types of hepatocytes using microarrays representing 54675 human transcripts. Among these, 16605 transcripts (30\% of total probes) were assigned as present ( $\mathrm{P}$ flag) for either all of the c-heps or all of h-heps, and $81.9 \%$ were expressed at similar levels $(<2$-fold difference) in the two types of hepatocytes.

The data for c-heps and h-heps (25YF, 28YM, 57YM, and $61 \mathrm{YF}$ ) and the $22 \mathrm{~h}$-tissues were clustered for 46336 probes. The cluster analysis showed that $6 \mathrm{c}$-heps formed a cluster, and the $4 \mathrm{~h}$-heps and h-liver formed another cluster, with these two clusters closest among the h-tissues (Supplementary Figure 1). PCA was performed for 46336 probes. PCA showed that the $6 \mathrm{c}$-heps and 4 h-heps were extremely close to each other, and that the h-liver was the closest to the cluster of the $6 \mathrm{c}$-heps and 4 h-heps among h-tissues (Figure 8a). We also performed cluster analysis using the 685 liver high signature probes and the 805 liver low signature probes for c-heps, h-heps, and the $22 \mathrm{~h}$-tissues. The cluster analysis demonstrated a close association among c-heps, h-heps, and h-liver (Figure 8b). Pearson's correlation coefficient was calculated for the expression levels of the liver-specific genes (685 probes), resulting in $0.812-0.909$ for h-heps $v s$ c-heps, $0.881-0.959$ for h-heps $v s$ h-heps, and 0.903-0.970 for c-heps vs c-heps.

The ratios of gene expression levels in the c-heps to those in the h-heps were compared using the data from the microarray and real-time qRT-PCR analyses. The expression levels of 17 genes were divided according to those of h-GAPDH. The microarray and real-time qRT-PCR data were well correlated (Spearman's correlation coefficient by rank test $=0.975$; Figure $8 \mathrm{c}$ ).

Moreover, of the 16605 transcripts, 436 transcripts (2.6\%) were twofold lower in expression in the c-heps than in the h-heps $(P<0.05$, two-sided Welch's $t$-test $)$. Of the same total, 197 transcripts $(1.2 \%)$ showed twofold greater expression in the c-heps than in the h-heps $(P<0.05$; Supplementary Table 3).

\section{DISCUSSION}

We morphometrically determined the structure of chimeric mouse livers, clarified the ratios of their component cells (heps, Kupffer cells, SECs, and stellate cells), and determined their morphological relationships through immunohistochemistry and electron microscopy. We recently reported that BrdU incorporation into h-heps was $\sim 6 \%$ at 2 or 3 weeks after transplantation, had decreased thereafter, and had dropped to $\sim 2 \%$ at 7 and 9 weeks after transplantation. At 11 weeks after transplantation, BrdU

Figure 5 Ultrastructure of the chimeric mouse livers as revealed by TEM (a-e) and SEM (f). The h-heps contained abundant glycogen and the presence of lipid droplets $(\mathbf{a}, \mathbf{d})$, whereas the mouse hepatocytes damaged by uPA expression showed an abundance of small granules (indicated by the arrow in the inset; $\mathbf{b}$ ). The mouse hepatocytes normalized by deletion of the uPA gene showed a normal structure (c). Bile canaliculi formed on the apical membranes of adjacent human hepatocytes, (d) and sinusoidal structures were observed on the basal cell membranes (a, d). A hepatic stellate cell containing lipid droplets was observed in the space of Disse (e). Fenestration was normally seen on SECs (f). Bar indicates $1 \mu \mathrm{m}$ in (a-c, e, f); $0.5 \mu \mathrm{m}$ in the squares of (b); and $5 \mu \mathrm{m}$ in (d). BC, bile canaliculi; BL, blood cell; HSC, hepatic stellate cell; L, lipid droplets; M, mitochondria; SD, sinusoid; SEC, sinusoidal endothelial cell. 

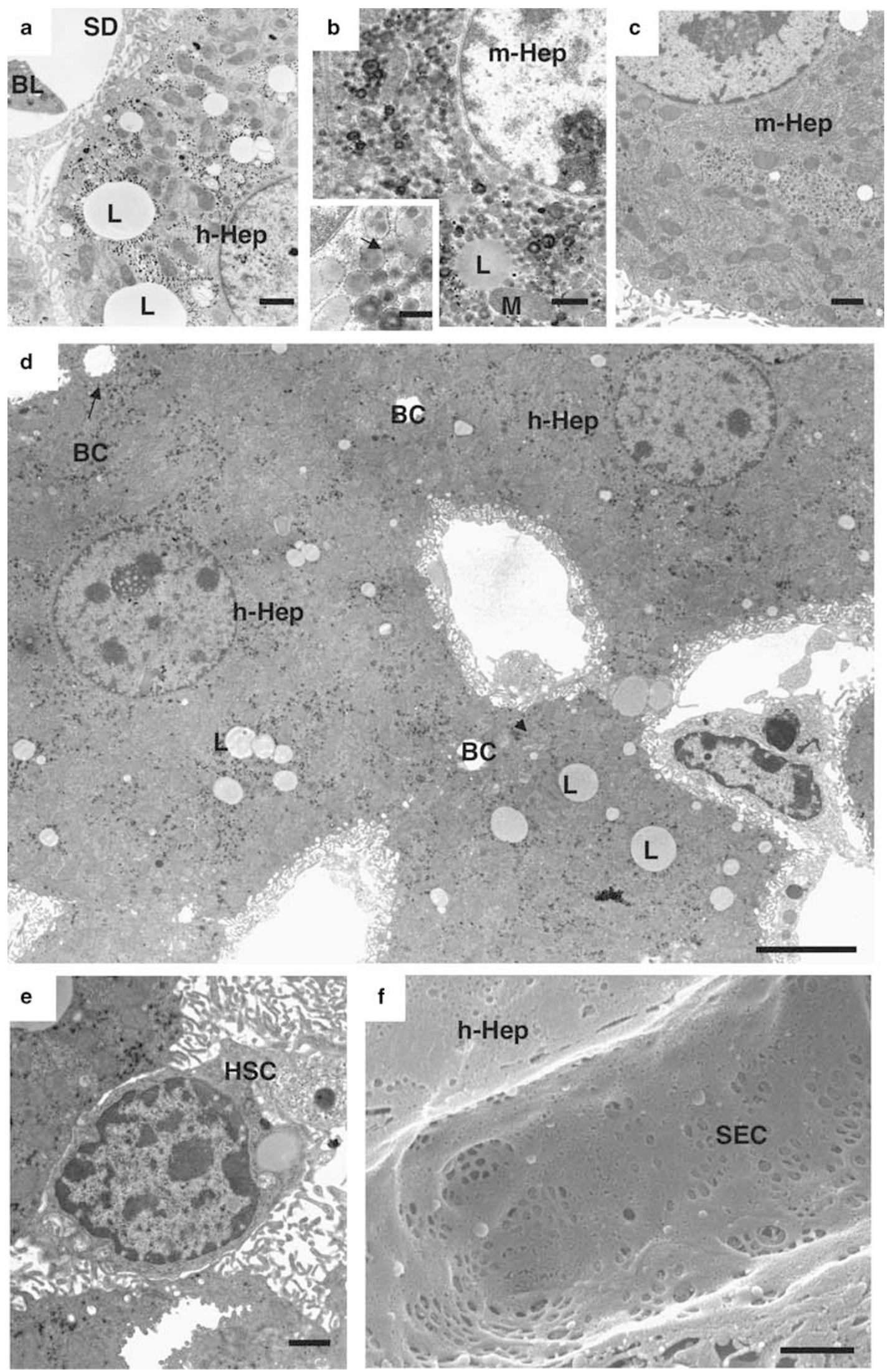
incorporation was $\sim 1 \%$, which was the same as the control level in adult SCID mouse livers. ${ }^{20}$ We also demonstrated that the liver weight to body weight ratios of chimeric mouse livers were $\sim 2$ times greater than those of normal mice when the transplants terminated proliferation. ${ }^{20}$ These results were considered to indicate hyperplasia of the h-heps, as no significant differences in cell size were observed. ${ }^{20}$ The mRNAs of proliferating h-heps contained lower levels of TGF- $\beta$ type I receptors (TGFBR1), TGFBR2, and activin A type IIA receptors (ACVR2A) than that of resting h-heps from human livers (normal levels), and these levels remained low when the transplants terminated proliferation. ${ }^{20}$ Therefore, we suggested that the chimeric mouse livers terminated their growth because of contact inhibition of h-heps or other mechanisms without the TGF- $\beta$ signaling pathway. From these data, we determined that the h-heps in the chimeric mouse livers were not completely in the G0 stage. The h-heps in the chimeric mouse livers showed twin-cell plates, indicating that some of the proliferating features continued as in regenerating or neonatal livers. ${ }^{20}$ In the present study, the morphometric analysis revealed similar HSC ratios in chimeric and SCID mice but an h-hep ratio in the chimeric mouse livers that was twofold higher than that in the SCID mouse liver, corresponding to the twin-cell plates in the chimeric mouse liver. The liver blood flow rate was similar between the SCID and chimeric mice. On the other hand, sinusoidal blood flow in the chimeric mouse was approximately one-half of that in the SCID mouse, probably because of the enlargement of the chimeric mouse liver. However, no disorders in the microcirculation of the chimeric mouse liver were observed. The oxygen consumption rate of hepatocytes is known to be negatively correlated with the average body weights of different species. ${ }^{23}$ Using a formula showing the relationship between resting hepatocyte oxygen consumption $(y)$ and body weight $(x)(y=7.09 x),{ }^{23}$ the ratio of the hepatocyte oxygen consumption of mouse (body weight: $0.02 \mathrm{~kg}$ ) to that of human (body weight: $60 \mathrm{~kg}$ ) is $\sim 5$. In the present study, the oxygen consumption of h-heps was $1 / 4.5$ of m-heps, which was very close to the above calculated rate.

After partial hepatectomy, the division of heps resulted in twin-cell plates, and the heps subsequently showed hypoxia. ${ }^{24}$ HIF-1 induction induced expression of downstream genes, such as VEGF and TGF- $\beta 3 .^{24}$ The author suggested that hypoxia of the heps induced reconstruction from twin-cell plates into single-cell plates by growth of the SECs. ${ }^{24}$ hHIF- $1 \alpha$, hVEGF, and hTGF- $\beta 3$ mRNA expression levels in the chimeric mouse livers were lower than those in human livers by real-time qRT-PCR (data not shown). In the present study, no evidence was found that the h-heps showed hypoxia in the chimeric mouse liver. This finding suggests that the h-heps in the chimeric mice may not be hypoxic, even in the twin-cell plates, because of low oxygen consumption in these cells.
With electron microscopic observation, we were able to easily distinguish $\mathrm{h}$-heps from $\mathrm{m}$-heps in the white areas because of the highly expressed uPA gene. The h-heps showed abundant glycogen and large lipid droplets, whereas m-heps in the white areas had abundant small vesicles in the cytoplasm. Distinguishing h-heps from $\mathrm{m}$-heps in the red areas was difficult because of the deletion of the uPA gene. M-heps retained quantities of glycogen particles, and careful observation of $\mathrm{m}$-heps in the red areas revealed prominent peroxisomes and fewer lipid droplets in the uPA-gene-deleted $\mathrm{m}$-heps than in the h-heps. On the other hand, very few peroxisomes were present in the h-heps. Junctional complexes and bile canaliculi were frequently observed between h-heps in the chimeric mouse livers. In rare cases, bile canaliculi were formed between h-heps and m-heps. In the peripheral cytoplasmic areas of two adjacent h-heps or a h-hep and a m-hep, abundant microvilli projected into the intercellular clefts on the lateral aspects of the hepatocytes. These characteristic morphological features have been frequently observed in regenerating livers. ${ }^{25}$ The formation of junctional complexes and bile canaliculi between $\mathrm{m}$-heps and h-heps is an important finding that demonstrates the ability of the mouse bile duct system to extract bile produced by the h-heps. Electron microscopic examination further revealed that SECs and stellate cells existed normally along hepatic cell plates. Fenestration was observed on the SECs in the chimeric mouse livers. These results indicate that chimeric mouse livers showed twin-cell plates like those often seen in regenerating and neonatal livers, whereas h-heps and $\mathrm{m}$-HSCs were normally reconstructed in the chimeric mouse livers.

The present study is the first to compare the gene expression patterns of c-heps and h-heps using microarray analysis. Approximately $82 \%$ were expressed at similar levels $(<2$-fold difference) in the two types of hepatocytes. There was the possibility that mouse transcripts were also included as cDNAs hybridized in the currently adopted microarray assay. Our previous study indicated that the RI represented a lower estimation of the real h-hepatocyte purity in hepatocyte preparations because $\mathrm{m}$-heps were often lost during collagenase digestion because of fragility against the enzyme. The correct h-hepatocyte purity in the c-heps was $90.8 \pm 6.4 \% \quad(n=10) .{ }^{17}$ We further checked the crosshybridization of the cDNAs of the mouse livers and found that 5643 of $54675(10.3 \%)$ transcripts were positive. As a whole, the presence of $\mathrm{m}$-heps in the c-heps at $<10 \% \operatorname{did}$ not affect the microarray assays in the present study. ${ }^{17}$ PCA and cluster analyses showed that the gene expression patterns of the c-heps were extremely similar to those of the h-heps. These data support our previous finding that c-heps retain phenotypes similar to those of h-heps, including P450 (CYP), phase II enzymes, and transporters. ${ }^{2-4}$ The c-hep samples and h-hep samples are clearly distinct with the 'normal' liver. One possible explanation is that the normal liver tissue contains both h-heps and h-HSCs. Using Fisher's test, we also analyzed 46336 probes that were assigned as 

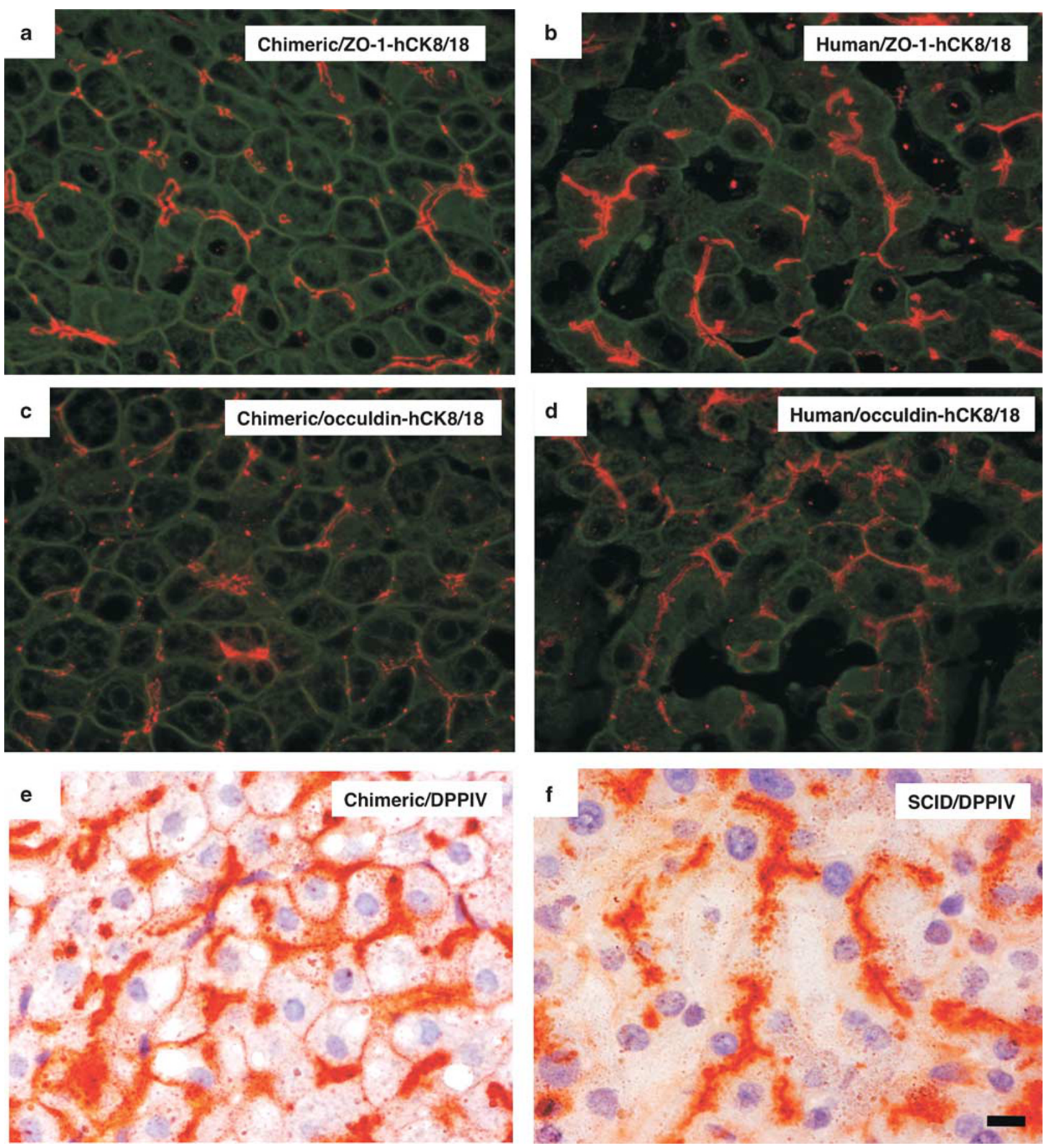

Figure 6 Immunohistochemistry of proteins related to cell adherence in human and chimeric mouse livers, and enzyme histochemistry of DPPIV in SCID and chimeric mouse livers. 9MM-chimeric mouse and human liver sections were stained with ZO-1 (a, b) and occludin (c, d) antibodies and stained for DPPIV $(\mathbf{e}, \mathbf{f})$. These proteins were located between hepatocytes in the chimeric mouse livers $(\mathbf{a}, \mathbf{c})$ and the human livers $(\mathbf{b}$, d). Fewer occludin deposits were observed in the chimeric mouse livers (c) than in the human livers (d). DPPIV-positive signals were located on the basal membranes in the SCID mice (f), but were also observed at the peripheries of human hepatocytes in chimeric mice (e). Bar $=10 \mu \mathrm{m}$. Magnification is the same in all panels.

positive ( $\mathrm{P}$ flag) for at least one of the flags in any of the c-heps, h-heps, or 22 h-tissues (Supplementary Table 4). The overlap $P$-values and odds ratio were determined between the liver signature probes and the probes of the h-heps with signals more than 2 times higher or lower compared with the average signals of all the tissues. The number of overlap probes were found to be 539-642 among 685 probes in the liver high expression signature, and the overlap $P$-values in the liver high 

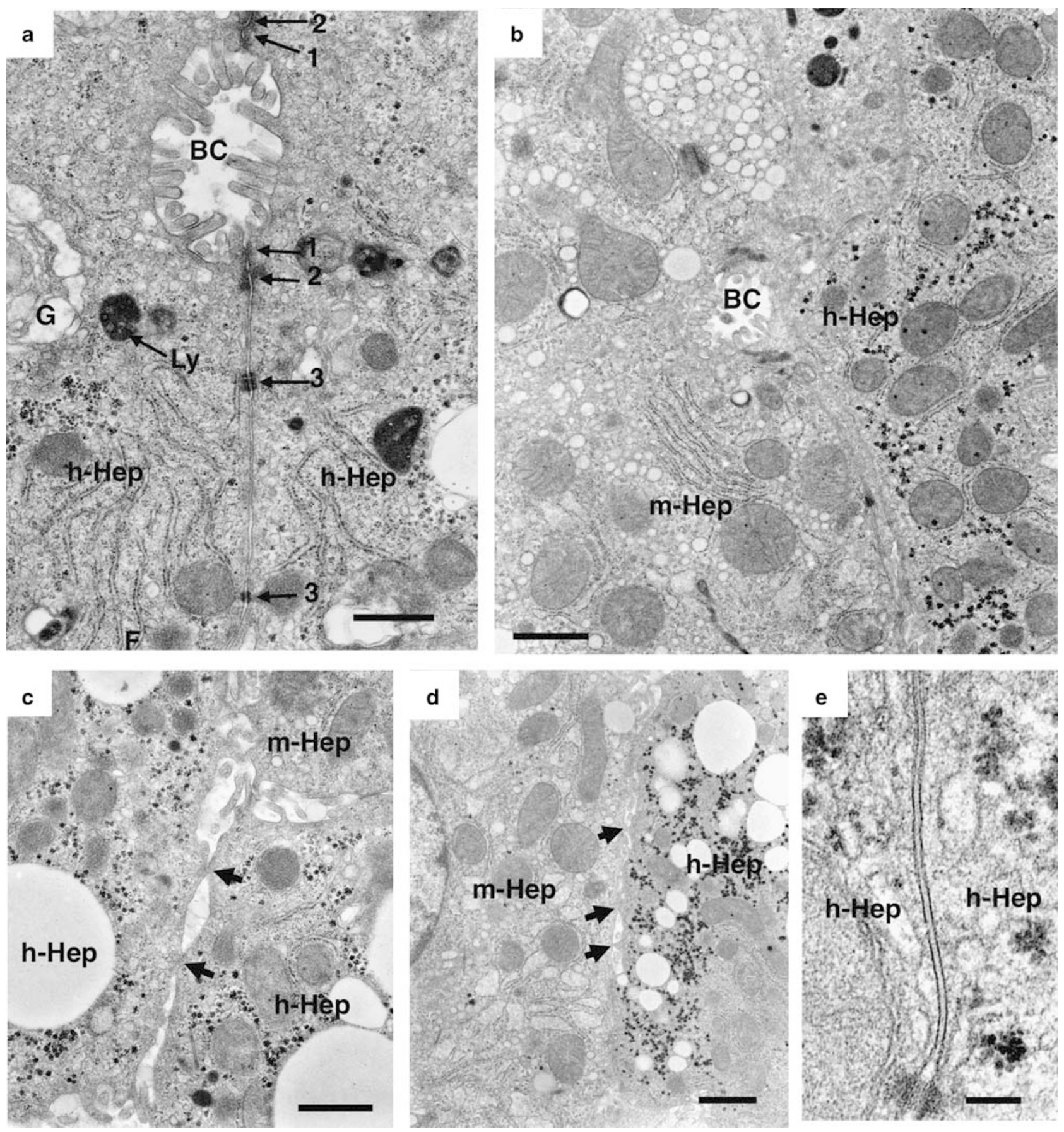

Figure 7 Ultrastructure of cell-cell contact in the chimeric mouse livers. Bile canaliculi were observed between adjacent h-heps (a) and between $\mathrm{h}$ - and $\mathrm{m}$-heps (b) in the 4YF-chimeric mouse liver, as well as junctional complexes (tight junction, 1; adherence junction, 2; desmosome, 3). In the peripheral cytoplasmic areas of groups formed by two adjacent human hepatocytes and one $\mathrm{m}$-hep, many microvilli projected into the intercellular clefts on the lateral aspects of the hepatocytes (c). We judged that the left and right cells in (b) are m-hep damaged by uPA expression and h-hep, respectively, because many membrane-limited granules are scattered throughout the cytoplasm in the left cell and the presence of abundant glycogen particles is observed in the cytoplasm in the right cell. A small number of cone-like cytoplasmic processes made contact with neighboring cells (c, d). Gap junctions were frequently observed between human hepatocytes (e). Bar $=0.5 \mu \mathrm{m}$ in (a); $1 \mu \mathrm{m}$ in (b, c, d); and $0.1 \mu \mathrm{m}$ in (e). BC, bile canaliculi; G, Golgi complex; Ly, lysosome.

expression signature were $<2.2 \times 10^{-16}$. The number of overlap probes were 153-485 among 805 probes in the liver low expression signature and were fewer than in the liver high expression signature; however, the overlap $P$-values in the liver low expression signature were also $<2.2 \times 10^{-16}$. From these data, we observe that the c-hep samples, h-hep samples, 


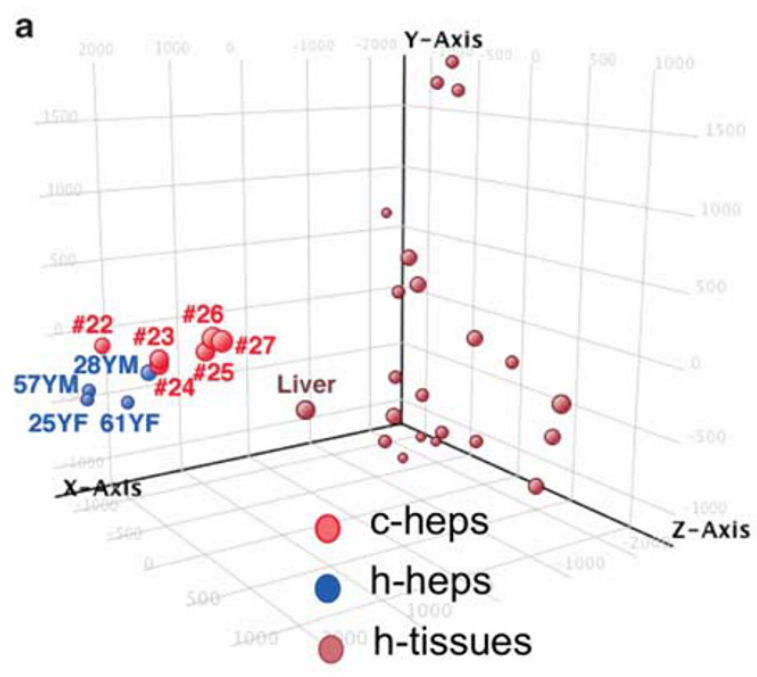

b
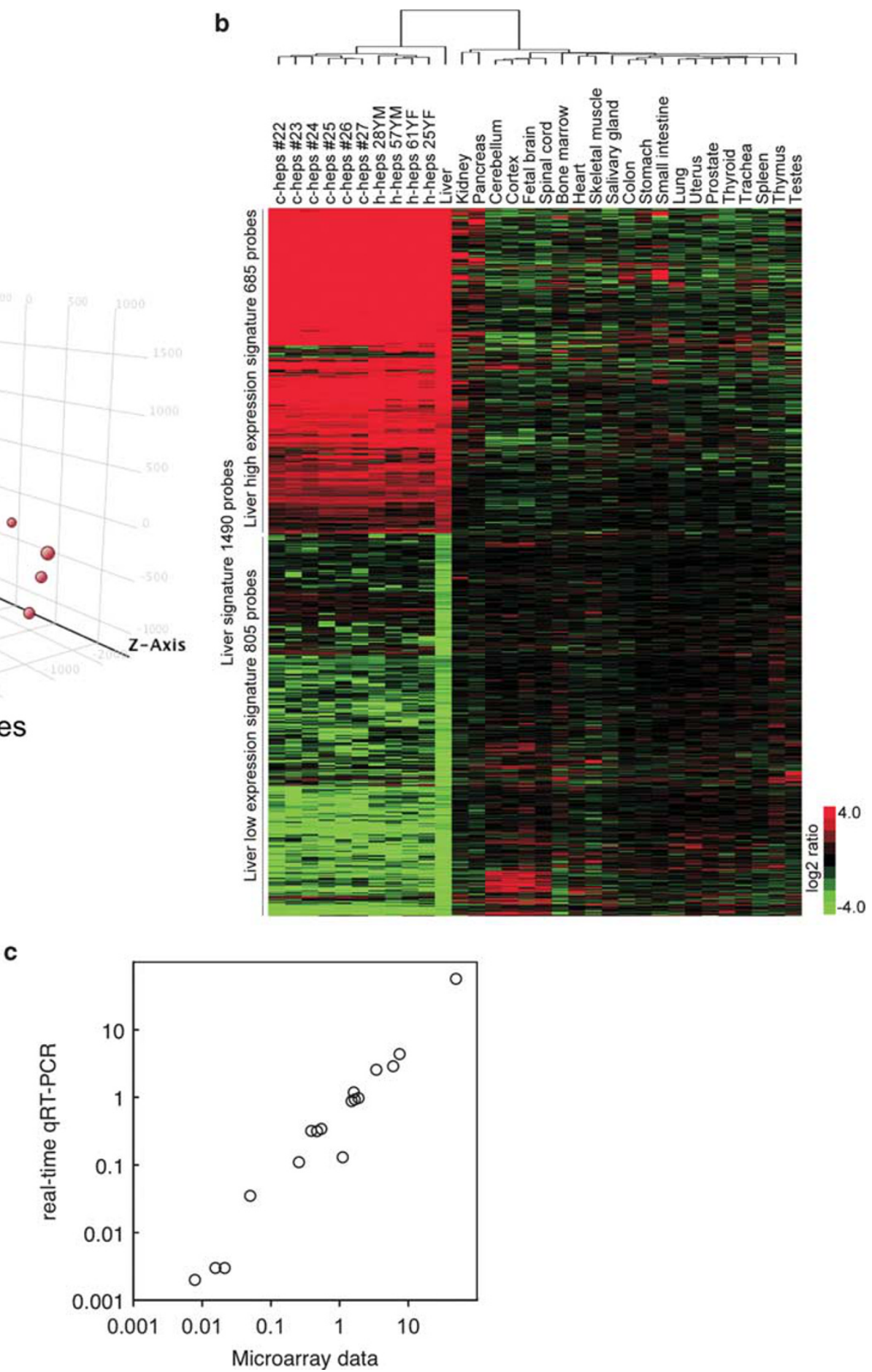

Figure 8 Similarity of gene expression between c-heps and h-heps. (a) PCA of c-heps, h-heps, and 22 h-organs or tissues. (b) Cluster analysis using the 685 liver high signature probes and the 805 liver low signature probes for c-heps, h-heps, and $22 \mathrm{~h}$-tissues. (c) Comparison of the gene expression profiles of chimeric and human hepatocytes using microarray and real-time qRT-PCR analyses. The ratios of gene expression in c- and h-heps, as obtained from the microarray analysis and real-time qRT-PCR, were compared for 17 genes. These data were well correlated.

and liver are highly similar, particularly in the liver high signature probes, but the differences in the expression levels of the liver low signature probes resulted in the clear distinction of the c-hep samples and h-hep samples with the 'normal' liver.
Microarray analysis showed that 191 (170 genes) and 436 (320 genes) probes were significantly ( $>2$ times) higher and lower, respectively, in c-heps than in h-heps. These genes may be up- and down-regulated in the chimeric mouse livers, probably by mismatched receptor-ligand combinations 
Table 3 Blood chemistry of chimeric, uPA/SCID, and uPA (wt/wt)/SCID mice

\begin{tabular}{|c|c|c|c|c|c|c|}
\hline Sex & & Male & Female & $\begin{array}{l}\text { uPA/SCID } \\
\text { Male }\end{array}$ & $\begin{array}{l}\text { SCID } \\
\text { Male }\end{array}$ & $\begin{array}{l}\text { Human (reference value, } \\
\text { Male and female) }\end{array}$ \\
\hline Age (w) & & $13.4 \pm 0.9$ & $12.0 \pm 1.9$ & $12.0 \pm 0.0$ & $11.0 \pm 0.0$ & - \\
\hline Body weight & & $17.3 \pm 2.3$ & $17.6 \pm 1.7$ & $17.4 \pm 0.3$ & $27.3 \pm 0.9$ & - \\
\hline GOT & $\mathrm{U} / \mathrm{I}$ & $214.6 \pm 75.1^{* * a}$ & $126.8 \pm 72.5$ & $367.6 \pm 107.7^{* * \mathrm{~b}}$ & $44.0 \pm 23.3$ & $20-48^{c}$ \\
\hline GPT & $\mathrm{U} / \mathrm{I}$ & $98.6 \pm 16.8^{* a}$ & $58.6 \pm 18.0$ & $176.4 \pm 28.9^{* * \mathrm{~b}}$ & $44.3 \pm 37.7$ & $10-40^{c}$ \\
\hline GGT & $\mathrm{U} / \mathrm{I}$ & $23.0 \pm 4.6^{* * a}$ & $15.0 \pm 3.9$ & $1.2 \pm 0.4$ & $3.7 \pm 4.6$ & $0-30^{c}$ \\
\hline $\mathrm{CHE}$ & $\mathrm{U} / \mathrm{I}$ & $396.8 \pm 80.2^{* * a}$ & $282.2 \pm 34.1$ & $42.6 \pm 8.0^{* * \mathrm{~b}}$ & $18.0 \pm 5.2$ & $660-1620^{d}$ \\
\hline BUN & $\mathrm{mg} / \mathrm{dl}$ & $27.3+4.0$ & $23.2 \pm 4.6$ & $24.6 \pm 9.1$ & $25.3 \pm 3.9$ & $8-23^{c}$ \\
\hline TBIL & $\mathrm{mg} / \mathrm{dl}$ & $0.4 \pm 0.1$ & $0.4 \pm 0.0$ & $0.5 \pm 0.2$ & $0.6 \pm 0.5$ & $0.3-1.2^{c}$ \\
\hline GLU & $\mathrm{mg} / \mathrm{dl}$ & $154.6 \pm 21.6$ & $148.8 \pm 31.9$ & $116.4 \pm 30.5$ & $154.7 \pm 14.2$ & $70-110^{c}$ \\
\hline ALB & $\mathrm{g} / \mathrm{dl}$ & $3.0 \pm 0.4^{* a}$ & $2.4 \pm 0.1$ & $1.7 \pm 0.3$ & $2.2 \pm 0.3$ & $3.5-5.0^{c}$ \\
\hline TP & $\mathrm{g} / \mathrm{dl}$ & $5.0 \pm 0.7$ & $4.2 \pm 0.3$ & $3.5 \pm 0.7^{* c}$ & $5.3 \pm 0.6$ & $6.0-8.0^{c}$ \\
\hline
\end{tabular}

${ }^{a}$ Chimeric mouse (male) vs SCID mouse (male).

buPA/SCID mouse (male) vs SCID mouse (male).

${ }^{\mathrm{c}}$ Reference number. ${ }^{21}$

${ }^{\mathrm{d}}$ Reference number. ${ }^{22}$

${ }^{*} P<0.05,{ }^{* *} P<0.01$

because of species differences. For example, h-heps were considered to be deficient in growth hormone $(\mathrm{GH})$ because the hGH receptor (hGHR) was unresponsive to mouse $\mathrm{GH}^{26}$ Human insulin-like growth factor 1 (IGF-1) was undetectable in chimeric mouse sera. ${ }^{17,27}$ We recently identified 4 downregulated and 14 upregulated genes in the chimeric mouse livers when they were treated with hGH. ${ }^{17}$ In this microarray analysis, the expression level of fatty acid desaturase 1 (FADS1, c-heps/h-heps ratio: 20.225) was significantly higher in c-hep than in h-heps (Supplementary Table 3). Meanwhile, the expression levels of IGF-1 (c-heps/h-heps ratio: 0.002), suppressors of cytokine signaling 2 (SOCS2; 0.012 and 0.030 ), nicotinamide $\mathrm{N}$-methyltransferase (NNMT; 0.025 and 0.019) chromosome 5 open reading frame 13 (C5orF13; 0.345), solute carrier family 16, member 1(SLC16A1; 0.485 and 0.432 ), steroid5 - $\alpha$-reductase, and $\alpha$-polypeptide 1 (SRD5A1; 0.486 and 0.461 ) were significantly lower in c-heps (Supplementary Table 3). Consequently, 1 of 170 genes and 6 of 320 genes were up- and down-regulated in c-heps, respectively, because of the lack of hGH in the sera.

Biochemical testing of the chimeric mouse sera revealed high GOT and GPT levels in chimeric and UPA/SCID mice, probably because of m-hep damage caused by uPA expression. ${ }^{2,28} \mathrm{CHE}$ was higher in chimeric mice than in UPA/SCID and SCID mice, which may be a result of the influence of typically higher h-hep CHE levels. Serum HDL-c levels are higher in mice than in humans, because mice lack cholesterol ester transport proteins that convert HDL-c to LDL-c. ${ }^{29}$ HDL-c was lower in the chimeric mice than in the uPA/SCID mice, SCID mice, and humans. At present, the reason for the low HDL-c levels in the chimeric mice is not clear. Further investigations are needed to resolve this question.

We conclude that the chimeric mouse livers showed nearly normal morphology and expressed most genes at similar levels as normal human livers.

Supplementary Information accompanies the paper on the Laboratory Investigation website (http://www.laboratoryinvestigation.org)

\section{ACKNOWLEDGEMENTS}

A part of this research was supported by the Yoshizato Project of the Cooperative Link of Unique Science and Technology for Economic Revitalization (CLUSTER), Japan. We thank $Y$ Yoshizane and R Inoue from PhoenixBio, and H Kohno, Y Matsumoto, and S Nagai from CLUSTER for their technical assistance. We also thank H Duimel, University Maastricht, for preparation of the SEM specimen.

\section{DISCLOSURE/CONFLICT OF INTEREST}

The authors declare no conflict of interest.

1. Dalvie $\mathrm{D}$, Obach RS, Kang $\mathrm{P}$, et al. Assessment of three human in vitro systems in the generation of major human excretory and circulating metabolites. Chem Res Toxicol 2009;22:357-368. 
2. Tateno C, Yoshizane Y, Saitou N, et al. Near completely humanized liver in mice shows human-type metabolic responses to drugs. Am J Pathol 2004;165:901-912.

3. Katoh M, Matsui T, Okamura $\mathrm{H}$, et al. Expression of human phase I enzymes in chimeric mice with humanized liver. Drug Metab Dispos 2005;33:1333-1340.

4. Nishimura $\mathrm{M}$, Yoshitsugu $\mathrm{H}$, Yokoi $\mathrm{T}$, et al. Evaluation of mRNA expression of human drug-metabolising enzymes and transporters in chimeric mouse with humanized liver. Xenobiotica 2005;35: 877-890.

5. Shi J, Fujieda H, Kokubo $Y$, et al. Apoptosis of neutrophils and their elimination by Kupffer cells in rat liver. Hepatology 1996;24: 1256-1263.

6. Warren A, Le Couteur DG, Fraser R, et al. T lymphocytes interact with hepatocytes through fenestrations in murine liver sinusoidal endothelial cells. Hepatology 2006:44:1182-1190.

7. Sato $\mathrm{Y}$, Yamada $\mathrm{H}$, Iwasaki $\mathrm{K}$, et al. Human hepatocytes can repopulate mouse liver: histopathology of the liver in human hepatocytetransplanted chimeric mice and toxicologic responses to acetaminophen. Toxicol Pathol 2008;36:581-591.

8. Meuleman P, Libbrecht L, De Vos R, et al. Morphological and biochemical characterization of a human liver in a uPA-SCID mouse chimera. Hepatology 2005;41:847-856.

9. Nonaka H, Tanaka M, Suzuki K, et al. Development of murine hepatic sinusoidal endothelial cells characterized by the expression of hyaluronan receptors. Dev Dyn 2007;236:2258-2267.

10. Katayama S, Tateno C, Asahara T, et al. Size-dependent in vivo growth potential of adult rat hepatocytes. Am J Pathol 2001;158. 97-105.

11. Ban D, Kudo A, Sui S, et al. Decreased Mrp2-dependent bile flow in the after-warm ischemic rat liver. J Surg Res 2009;153:310-316.

12. Mabuchi $A$, Wake $K$, Marlini $M$, et al. Protection by glycyrrhizin against warm ischemia-reperfusion-induced cellular injury and derangement of the microcirculatory blood flow in the rat liver. Microcirculation 2009;16:364-376

13. Wisse $\mathrm{E}$, Braet $\mathrm{F}$, Duimel $\mathrm{H}$, et al. Fixation methods for electron microscopy of human and other liver. World J Gastroentero 2010;16:2851-2866.

14. Seglen PO. Preparation of isolated rat liver cells. Methods Cell Bio 1976;13:29-83.

15. Bolstad BM, Irizarry RA, Astrand $\mathrm{M}$, et al. A comparison of normalization methods for high density oligonucleotide array data based on variance and bias. Bioinformatics 2003;19:185-193.
16. Eisen MB, Spellman PT, Brown PO, et al. Cluster analysis and display of genome-wide expression patterns. Proc Natl Acad Sci USA 1988;95:14863-14868

17. Tateno C, Kataoka M, Utoh $\mathrm{R}$, et al. Growth hormone-dependent pathogenesis of human hepatic steatosis in a novel mouse mode bearing a human hepatocyte-repopulated liver. Endocrinology 2011;152:1479-1491.

18. Asahina $K$, Sato $H$, Yamasaki $C$, et al. Pleiotrophin/HB-GAM as a mitogen of rat hepatocytes and its role in regeneration and development of liver. Am J Pathol 2002;160:2191-2205.

19. Benjamini Y, Hochberg Y. Controlling the false discovery rate: a practical and powerful approach to multiple testing. J Roy Statist Soc Ser B 1995;57:289-300.

20. Utoh R, Tateno C, Kataoka M, et al. Hepatic hyperplasia associated with discordant xenogeneic parenchymal-nonparenchymal interactions in human hepatocyte-repopulated mice. Am J Pathol 2010;177:654-665.

21. Michael L. Laboratory Medicine: The Diagnosis in the Clinical Laboratory, New York, 2010.

22. Jensen FS, Skovgaard LT, Viby-Mogensen J. Identification of human plasma cholinesterase variants in 6,688 individuals using biochemical analysis. Acta Anaesthesiol Scand 1995;39:157-162.

23. Porter RK, Brand MD. Causes of differences in respiration rate of hepatocytes from mammals of different body mass. Am J Physiol 1995;269:R1213-R1224.

24. Maeno $\mathrm{H}$, Ono $\mathrm{T}$, Dhar DK, et al. Expression of hypoxia inducible factor-1alpha during liver regeneration induced by partial hepatectomy in rats. Liver Int 2005;25:1002-1009.

25. Tomoyori T, Ogawa K, Mori M, et al. Ultrastructural changes in the bile canaliculi and the lateral surfaces of rat hepatocytes during restorative proliferation. Virchows Arch B Cell Pathol Incl Mol Pathol 1983;42: 201-211.

26. Souza SC, Frick GP, Wang $X$, et al. A single arginine residue determines species specificity of the human growth hormone receptor. Proc Natl Acad Sci USA 1995;92:959-963.

27. Masumoto N, Tateno C, Tachibana A, et al. GH enhances proliferation of h-heps grafted into immunodeficient mice with damaged liver. J Endoclinol 2007;194:529-537.

28. Sandgren EP, Palmiter RD, Heckel JL, et al. Complete hepatic regeneration after somatic deletion of an albumin-plasminogen activator transgene. Cell 1991;66:245-256.

29. Dinchuk J, Hart J, Gonzalez G, et al. Remodelling of lipoproteins in transgenic mice expressing human cholesteryl ester transfer protein. Biochim Biophys Acta 1995;1255:301-310. 